\title{
Task scheduling system for UAV operations in indoor environment
}

\author{
Yohanes Khosiawan ${ }^{1}$ Youngsoo Park ${ }^{2}$ Ilkyeong Moon ${ }^{2,3} \cdot$ Janardhanan Mukund Nilakantan ${ }^{1}$. \\ Izabela Nielsen ${ }^{1}$
}

Received: 23 February 2016/Accepted: 21 February 2018/Published online: 3 March 2018

(C) The Author(s) 2018. This article is an open access publication

\begin{abstract}
The application of unmanned aerial vehicle (UAV) in indoor environment is emerging nowadays due to the advancements in technology. UAV brings more space flexibility in an occupied or hardly accessible indoor environment, e.g. shop floor of manufacturing industry, greenhouse, and nuclear powerplant. UAV helps in creating an autonomous manufacturing system by executing tasks with less human intervention in a time-efficient manner. Consequently, a scheduler is an essential component to be focused on; yet the number of reported studies on UAV scheduling has been minimal. This work proposes a mathematical model of the problem and a heuristic-based methodology to solve it. To suit near real-time operations, a quick response towards uncertain events and a quick creation of new high-quality feasible schedule are needed. Hence, the proposed heuristic is incorporated with particle swarm optimization algorithm to find a near optimal schedule in a short computation time. This proposed methodology is implemented into a scheduler and tested on a few scales of datasets generated based on real flight demonstrations. Performance evaluation of scheduler is discussed in detail, and the best solution obtained from a selected set of parameters is reported.
\end{abstract}

Keywords Indoor UAV system · Particle swarm optimization $\cdot$ Scheduling · Autonomous system

\section{Introduction}

In the recent years, usages of unmanned aerial vehicles (UAVs) have been increasingly prominent for various applications such as surveillance, logistics, and rescue missions.

Izabela Nielsen

izabela@mp.aau.dk

Yohanes Khosiawan

yok@mp.aau.dk

Youngsoo Park

simulacrum@snu.ac.kr

Ilkyeong Moon

ikmoon@snu.ac.kr

Janardhanan Mukund Nilakantan mnj@mp.aau.dk

1 Department of Materials and Production, Aalborg University, 9220 Aalborg, Denmark

2 Department of Industrial Engineering, Seoul National University, Seoul 08826, Republic of Korea

3 Institute for Industrial Systems Innovation, Seoul 08826, Republic of Korea
UAVs are very useful for monitoring activities which are tedious and dangerous for human intervention [33]. Most of the UAVs commercially available so far have the capability of operating in an outdoor environment [1, 47]. Previously, UAV applications used to be limited for only military purposes, but nowadays the situation has changed [8]. UAV emerges as a viable, low-cost technology [42] for use in various indoor applications [38]. With the advancements in technology, the scope of the UAV application in indoor environment becomes a rising interest among different industries. UAVs can be useful for executing multiple tasks in indoor environments of manufacturing and service industries (e.g. hospital, green house, and wind turbine manufacturer), which has not been reported so far. UAVs can be equipped with a high-resolution camera to monitor the indoor environment, and UAVs can support material handling by transporting different parts/materials between locations in an indoor environment. Despite various challenges and growing interests in UAV application in indoor environment, the research related to this area is at an early stage.

There are many components involved when a UAV system is implemented in an indoor environment, e.g. robust wireless communication, three-dimensional 
trajectory data, precise UAV control, and schedule (which reflects the required commands for UAV control). A schedule creation mainly aims at assigning tasks to UAVs in a manner that efficiently utilizes the available UAVs. There is a minimal number of reported works on UAV applications in indoor environment [23]. In this paper, tasks and other (on-demand) actions (i.e. recharge, hover, and wait-on-ground) are assigned to the UAVs at different execution times. This assignment is done in regard to the constraints exposed by the $3 \mathrm{D}$ positioning system, where precise position coordinates and position occupation management over time are crucial. This is the essential gap between the problem faced in this study and the state of the art of UAV applications in indoor environment. To obtain an optimum schedule with a branch-and-bound-based method, an exponentially growing computation time is anticipated. This condition entails the heuristic-based approach, whose nature obtains a good quality feasible solution in a short computation time. The main contributions of this paper are mentioned as follows.

1. Designed a system architecture for UAV applications in indoor environment.

2. Developed a formal description of the problem in the form of a mathematical model.

3. Developed a methodology which includes:

- a heuristic based on the earliest available time algorithm for task scheduling with an objective of minimizing makespan,

- an incorporation of the proposed heuristic with particle swarm optimization (PSO) algorithm to obtain a feasible solution in a short computation time.

4. Tested and evaluated performance of the proposed methodology using benchmark data generated based on real flight demonstrations at laboratory.

The remainder of the paper is structured as follows. Section 2 presents the literature survey, and Sect. 3 explains the problem and detailed framework of the proposed scheduling system. Section 4 describes the key elements involved in the implementation of PSO and the proposed methodology. Sections 5 and 6 discuss numerical experiments and results of the implemented methodology. Section 7 concludes the findings of this research.

\section{Literature review}

The essential key to successful UAV operations is a robust system of command and control [30]. A UAV control navigates the UAV's movement to have a seamless flight during the operations. The required navigation control is derived from a command which is provided by a command centre, referred as a scheduler in this study. This section gives a detailed summary of related studies which focus on UAV scheduling. Some researchers focused on developing the scheduling system for UAVs without considering travel time or distance restriction [50]. Authors in [50] developed a single-objective nonlinear integer programming model for solving a UAV scheduling problem which aims at allocating and maximizing the utilization of the available UAVs in an efficient manner. They tested the proposed model using a small sized problem for an outdoor environment.

Shima and Schumacher [43] developed scheduling methods for UAVs without any fuel limitation. A mathematical model which instructs a cooperative engagement with multiple UAVs was developed. It was addressed that assigning multiple UAVs to perform multiple tasks is an NP-hard combinatorial optimization problem. To obtain a feasible solution, a genetic algorithm was proposed. The problem mainly aims at assigning different tasks to different UAVs and consequently assigning the respective flying path to each UAV. From their experimental results, it is seen that genetic algorithm is efficient in providing real-time good quality feasible solutions. Kim et al. [25] proposed a mixed integer linear programme (MILP) model to formalize the problem of scheduling system of UAVs. In their model, trajectories or jobs are split into different pieces and are referred as split jobs. This method is useful when a UAV is not capable of performing the entire task within a single flight travel due to the fuel (or battery) capacity constraint. The authors benchmarked the performance of genetic algorithm against CPLEX solver and found out that genetic algorithm obtained feasible solutions in a reasonable computation time for the cases in which CPLEX cannot even solve.

In a further work, Kim and Morrison [24] proposed a modified receding horizon task assignment heuristic $\left(\mathrm{RHTA}_{d}\right)$. In the mentioned problem assumptions, UAV should complete the tasks within its fuel (battery) capacity and return to the base before the fuel runs out. The proposed MILP seeks to minimize the total system cost, which comprises travel and resource costs, and to ensure that every mission is provided at least one UAV at all times. The formulated MILP determines the types and numbers of UAVs, as well as locations and numbers of stations. The developed RHTA $_{d}$ was then compared with branch and bound algorithm to solve the referred problem.

Weinstein and Schumacher [48] developed a UAV scheduling problem based on the inputs of vehicle routing problem which considers time window constraint. The vehicle routing problem is solved through MILP (using CPLEX and self-implemented branch and bound algorithm) with a target to find a global optimal schedule. 
Kim et al. [26] proposed a scheduling model for $n$ tasks and $m$ UAVs each having a capacity limit of $q$ in a hostile environment. The proposed model aims at minimizing the cost due to the operation time and risk exposed. An MILP formulation is proposed first which exactly solves the problem and later they proposed four alternative MILPs which are computationally less intensive. The proposed model was highly complicated with huge number of variables and constraints, making them impractical for application. Improvements to the model were proposed in [3] which minimized the number of variables and constraints.

A few works on establishing a persistent UAV service have been addressed in $[5,24,30,46]$, which concentrate on enabling a long-duration task execution. However, none of these works focused on scheduling multiple tasks at multiple positions executed by multiple UAVs. Furthermore, UAV operations in indoor environment make the complexity of scheduling problem higher and there is a minimal work reported in this area. Some aforementioned works on persistent UAV service are for surveillance purpose in indoor environment, but with no or minimal obstacles. This research focuses on developing a methodology to assign different tasks to different UAVs in timeefficient manner for indoor environment, and there is a requirement of finding an exact schedule which allows the UAVs to fly autonomously. Scheduling system should react to uncertain events (e.g. UAV breakdown, fuel constraints) which may happen during UAV operations. Hence, there is a need of generating a fast feasible schedule. Generating schedules using MILP is not computationally viable, and metaheuristic is an alternative in such scenarios.

UAV scheduling is a complex problem, and researchers have utilized CPLEX (employing a proprietary method which incorporates branch \& bound and branch \& cut algorithm [29]), heuristics, and genetic algorithm to solve it. However, it could be postulated from the literature review that there has been no work reported on using metaheuristic algorithms to solve UAV scheduling problem. Various works have been reported in the literature where different metaheuristics are used to solve specific types of scheduling problems (e.g. job shop, flow shop, and cyclic scheduling problems) $[39,51]$ due to the NP-hard nature of these problems. Concept of job-shop scheduling problem (where jobs may be assigned to different sets of processors at particular times) and parallel machine scheduling (where tasks are assigned to a number of processors) can be classified as a part of UAV scheduling problem [14]. Studies on job-shop scheduling problem have been focused on solving different objective functions such as minimizing makespan, lateness, energy consumption, and maximizing utilization [6], wherein both job-shop and parallel machine scheduling problem are known as NPhard [14]. To a further extend, from the UAV control problem perspective, researchers have found it beneficial to employ metaheuristics such as differential evolution and its hybridization in [4, 28, 45] for identification of UAV. Researchers shift their focus towards metaheuristic as a popular way to address approaches on problems of this nature.

This paper proposes a methodology which includes an earliest available time (EAT) algorithm incorporated with particle swarm optimization (PSO) algorithm with an objective of minimizing the makespan. EAT constructs a schedule from a task sequence, which is the solution representation used in the PSO framework. PSO is a relatively new metaheuristic approach developed by Kennedy and Eberhart [21]. PSO is one of the prominent evolutionary computation methods employed to solve scheduling problems [27], but it has not been used for the addressed UAV scheduling problem in this paper. The following section provides details of the problem addressed and the framework of the proposed system. This study is the real case of the presented preliminary study in [22], and some information is briefly reproduced to give clarity to the reader.

\section{Problem definition}

This section presents the details of UAV scheduling problem in indoor environment. Compared to outdoor environment, UAV application in indoor environment requires more constraints and precise control [30]. Thus, in this section, a framework of the UAV system components in indoor environment is designed in a systematical way. As a whole, Sects. 3.1-3.3 present a reference model [10], as a guide for various UAV applications in indoor environment. A reference model can be used to employ UAVs in various general systems by specifying the domain (environment), platform (UAV operation system - see Fig. 1), and the interface between them [17]. Afterwards, the architecture of UAV scheduling system and phase-based scheduling framework are presented. In Sect. 3.1, the UAV system in indoor environment is defined in three layers. In Sect. 3.2, the UAV scheduling system is presented. Finally, the designed scheduling framework is defined in Sect. 3.3.

\subsection{UAV system in indoor environment}

A three-layer architecture of an indoor UAV system is depicted in Fig. 1. Its concept is introduced in a preliminary work [22], which is now discussed in detail in regard to this pilot work. It is a combination of the logical representation of the UAV operations and the physical representation of the UAV environment. The respective three layers are described as follows. 
Fig. 1 Architecture of indoor UAV system

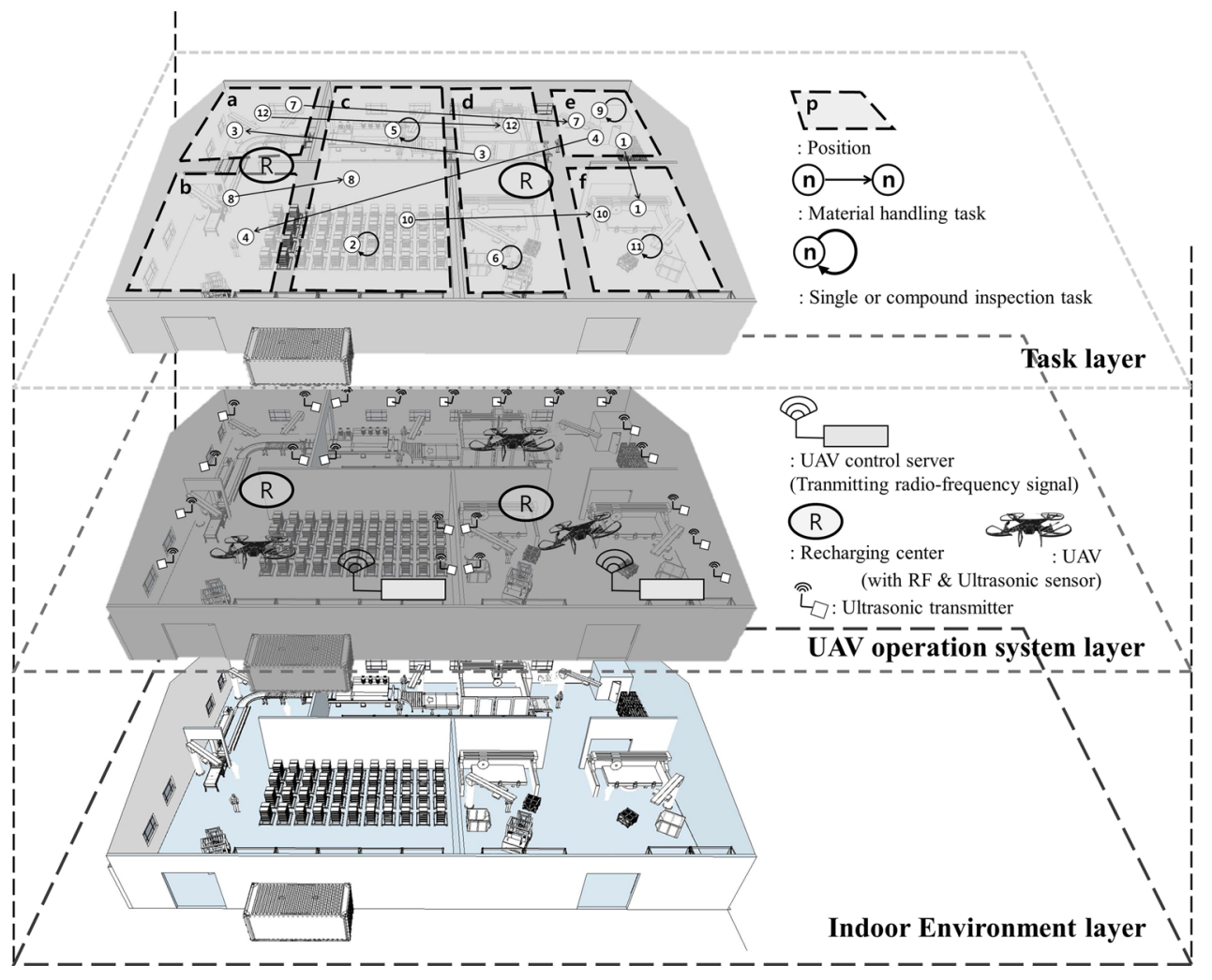

1. Indoor environment layer contains the infrastructure (e.g. machine, conveyer belt, assembly line) where the UAV system is implemented. The environment structure and the UAV operations performed in it can affect each other's requirements. For instance, a dedicated corridor for material handling task is defined in a highly occupied shop floor (i.e. occupied by machines and human labours) to suppress the safety risk. Other representations of task and environment might have different characteristics that affect each other. Furthermore, to avoid collision among UAVs and obstacles during the operations, the environment is segregated into zones, which practically indicate areas which are currently occupied by UAVs, permanent obstacles, and other (environmental) temporary obstacles (e.g. air turbulence due to gas pipe leak). This concept of zones will be incorporated in the future work. Currently, collision avoidance at a position is implemented by allowing only one UAV to occupy a position at a particular time. A proper investigation of real-time sensor-based collision avoidance by the UAV [13] will also be conducted to be considered in the scheduling system. Budiyono et al. [9] approached this issue with a kinect sensor which sends imaging data of the detection zone to an application, and it gives a warning message and avoidance command to the flight control system on the UAV. For a real-time application, an embedded computing unit can be put on the UAV to perform an immediate action to halt the flight and hover when a sudden obstacle is encountered-the scheduling system is then notified of such an event to take the appropriate measure towards the corresponding schedule.

2. UAV operation system layer consists of UAVs and other supporting entities for UAV operations in indoor environment. Ultrasonic transmitters are mounted in the indoor environment to establish a UAV positioning system. A UAV scheduling system plans the task executions and instructs the respective UAVs via a UAV control server. The UAV control server interacts with the UAVs through a radio frequency protocol. In case of lost communication link, the UAV is preprogrammed to fly back to a designated recharge station. Recharge station carries out an autonomic UAV recharge, where the UAV needs to land on a designated position.

3. Task layer contains actions for the UAVs to execute. A detailed information of each task (e.g. type of task, start and end position, and precedence relationship) needs to be defined. Start and end positions signify the origin (pickup position) and destination (release position) for material handling task, while they will be one 
(identical) inspection position for single and compound inspection tasks. A task may have precedence relationships, which means that it is only executed after specific tasks in a predecessor list are completed.

The proposed indoor UAV system operates multiple UAVs to execute multiple tasks. Tasks are nonpreemptive and exclusively assigned to one UAV. In Table 1, there are three types of task: (1) single inspection, (2) compound inspection, and (3) material handling task. A single inspection task consists of a flight to a specific position and an image capture with a built-in camera. A compound inspection task consists of multiple single inspections whose points of interest are located around one identical position. Material handling task consists of a pickup action, a flight to the release point, and a release action. This task is performed using a built-in equipment.

The UAVs considered in this work are identical multicopters with a built-in camera and a material handling equipment, which can handle the addressed types of task. The considered UAVs are capacitated; hence, a UAV can execute multiple tasks in one flight route to the limit of the battery capacity. They can also hover in the air or wait-onground at the predefined position. With the assumed proportional weight ratio of payload to UAV, the flight speed and the battery consumption of the UAV are constant. In the schedule, the dimension of the time is discrete. Task execution timestamp, other action execution timestamp (such as flight, hover, wait-on-ground, and recharge), and task execution time (time required to execute a task) are planned. The assumptions of the presented problem are defined as follows.

1. The system is deterministic; there is no uncertain event.

2. Execution of task is non-preemptive, thus not divisible into subtasks.

3. A task is executed by exactly one UAV.

4. Every task execution time is shorter than the flight time limit (based on the battery constraint).

5. Within the proportional payload level, UAV has a constant flight speed and battery consumption rate.

6. UAVs are equipped with built-in sensors for local collision avoidance mechanism at the paths.
7. In every flight, a capacitated UAV has a fixed amount of:

(a) flight time (in this work, it is up to $1200 \mathrm{~s}$ ) and

(b) recharge time (in this work, it is $2700 \mathrm{~s}$ ) at a designated recharge station.

8. The recharge time of $2700 \mathrm{~s}$ includes the time to travel from the waiting area (when the recharge slots are occupied) to the recharge station. This assumption is realistic when an automated (conveyer beltlike) mechanism places the UAV to the recharge slot without requiring it to fly from its waiting position.

9. Partial recharge is not allowed.

10. Multiple recharge stations exist.

\subsection{UAV scheduling system}

To execute tasks efficiently, a UAV scheduling system is needed to assign tasks to UAVs while considering the execution timestamps of all involved actions in a coherent manner (and correspondingly construct a schedule). Hence, it is an essential part of UAV operation system. In the UAV scheduling system, scheduler component interacts with task database, trajectory database, and UAV database. Task database stores detailed information (e.g. processing time, start and end positions, and precedence relationship) of the tasks to be executed.

Trajectory database provides a three-dimensional trajectory map (which includes waypoints, paths, and positions where tasks and recharges are held), including shortest possible routes between positions. A route in the high-level map has the total weight of the respectively comprised paths in the low-level map [22]. High-level map is required for reducing the solution space and computation time during the schedule generation, while low-level map is needed for translating the schedule to UAV-compatible instructions before they are sent to the UAVs.

\subsection{Phase-based scheduling framework}

Scheduler component works in phases, which are designed for abstraction of the schedule and map. Abstraction is needed to reduce the solution space and minimize the computation size. On the other hand, autonomously operated UAVs need a detailed and precise command, which is

Table 1 Task types

\begin{tabular}{lll}
\hline Task type & Action & Description \\
\hline Single inspection & Inspection & Capture an image at a designated position \\
Compound inspection & Inspections (more than one) & Capture images of points of interest around a position \\
Material handling & Pickup-flight-release & Transport a material from an origin to a destination position \\
\hline
\end{tabular}


enabled by the accurate physical three-dimensional map. Therefore, the scheduler component applies two levels of flight schedule and map. In accordance with the aforementioned trajectory data, there are low-level map and high-level map. Low-level schedule builds on a low-level map, which specifies detailed flight paths and timestamps of subtasks (actions). High-level schedule consists of (more highly abstracted) actions such as tasks (e.g. material handling task, inspection task), flight between tasks, hover, and wait-on-ground.

Phase-based scheduling framework consists of two phases: assignment and anti-collision refinement. From its introduction in the preliminary work [22], the framework has been improved in this work tailoring its solvability. In order to respond to uncertain events, scheduler component needs to find a feasible schedule in a short computation time. Hence, separating task assignment and detailed (paths) routing is required for reducing the computation size. Phase-based scheduling framework is presented in Fig. 2. Furthermore, the proposed scheduler tries to create a new schedule in a time-efficient manner which satisfies the corresponding constraints in regard to the occurred uncertain events.

The main contribution of this work is scoped as phase 1 . In phase 1, scheduler assigns tasks to UAVs. Timestamps for each UAV to start the assigned task, required flight, hover, wait-on-ground, and recharge are planned. The output of phase 1 is a high-level schedule of the UAVs.

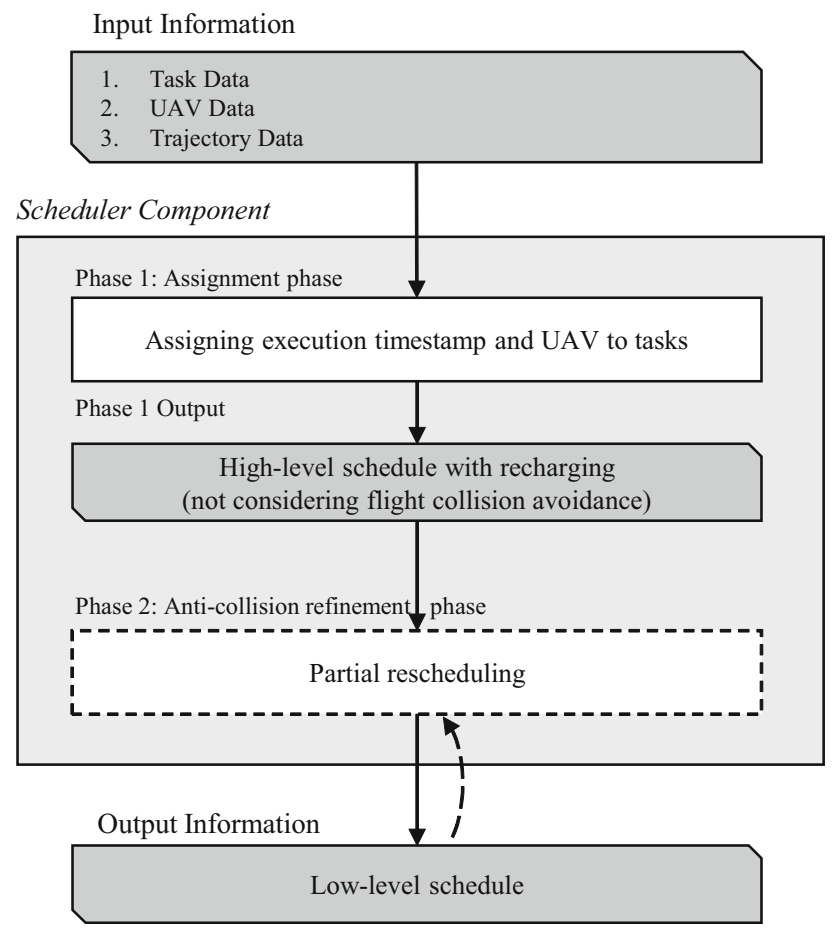

Fig. 2 Phase-based scheduling framework
Scheduler uses the timespan of the task executions and flights to calculate the battery usage and to avoid collision while handling tasks at the designated positions. A position is occupied by only one UAV at a time to avoid collision. This procedure is organized into a proposed heuristic which is depicted in Algorithm (2) later.

The output of phase 1 is then processed in the next phase due to the following reasons. First, for producing UAVcompatible instructions from the obtained (high-level) schedule, a translation to a low-level schedule is needed. Second, the output of phase 1 does not consider the possible collision caused by intersecting flight path. In phase 2 , the high-level schedule is divided into subactions, and the low-level schedule is derived. UAV operations in indoor environment tend to have less alternatives of deterministic routes between positions. Keeping this into consideration, the structure of the optimal schedule tends to be the same even when some delays are introduced during the schedule executions. An instruction from the schedule can be sent to the respective UAV if the currently monitored battery level is sufficient. If the battery constraint is violated, a (partial) rescheduling of the remaining tasks will be done. On the other hand, when one generates a path-collision-free schedule prior to the operations-with the sacrifice of a higher computation time, there is a high chance of deviation from the schedule during its execution (due to, for example, uncertain events from the indoor environment, UAV operation system, and the UAV). Hence, the planned phase 2 is computationally more adaptable in practice, and this will be addressed in the future work.

In phase 1, there are three types of input data: (1) task data, (2) UAV data, and (3) trajectory data [22]. Task data used in phase 1 consist of task identifier, start position, end position, processing time, and precedence relationship. Start and end positions are needed to calculate the respective task processing time. In addition, both are used by one UAV at a time to avoid collisions. Processing times are needed to calculate the task execution timestamps, and precedence relationships among tasks are used to check the current availability of each task. Table 2 is an example of the task data. UAV data consist of current state, position, and battery status of each UAV. Trajectory data used in phase 1 are built upon the travel time data between positions in the high-level map. The map is considered as a complete graph, and all the distances are calculated based on the shortest path between positions.

Table 3 is an example of the trajectory data containing 6 positions $(a-f)$ and 2 recharge stations $(R 1, R 2)$, based on the shortest flight time. This data example is used to illustrate how the proposed methodology works in Sect. 4. As the result, a schedule is generated-which comprises the task executions and other actions assigned to the UAVs. 
Table 2 Task data

\begin{tabular}{lllll}
\hline TaskID & Start position & End position & Processing time (s) & Precedents \\
\hline 1 & e & f & 243 & - \\
2 & c & c & 245 & - \\
3 & d & a & 719 & - \\
4 & e & b & 550 & 1 \\
5 & c & c & 235 & 2 \\
6 & d & d & 241 & 4 \\
7 & a & e & 478 & 4,5 \\
8 & b & c & 304 & 7 \\
9 & e & e & 395 & 6,8 \\
10 & c & f & 344 & 10 \\
11 & f & f & 270 & 3,6 \\
12 & a & d & 514 &
\end{tabular}

Table 3 Position and distance (in flight time unit) data

\begin{tabular}{llrrrrrrr}
\hline From/to & a & \multicolumn{1}{c}{ b } & \multicolumn{1}{l}{ c } & d & e & f & R1 & R2 \\
\hline a & 0 & 108 & 131 & 222 & 376 & 353 & 40 & 160 \\
b & 108 & 0 & 120 & 241 & 347 & 371 & 60 & 160 \\
c & 131 & 120 & 0 & 127 & 228 & 254 & 60 & 60 \\
d & 222 & 241 & 127 & 0 & 116 & 122 & 160 & 40 \\
e & 376 & 347 & 228 & 116 & 0 & 123 & 260 & 60 \\
f & 353 & 371 & 254 & 122 & 123 & 0 & 260 & 60 \\
R1 & 40 & 60 & 60 & 160 & 260 & 260 & 0 & 120 \\
R2 & 160 & 160 & 60 & 40 & 60 & 60 & 120 & 0 \\
\hline & & & & & & & &
\end{tabular}

The Gantt chart representation is depicted in Fig. 3, and the respective data are listed in Table 4. For instance, UAV1 performs a flight action from $R 1$ to $c$ at time $0-40$. It is followed by an inspection task (2) at $c$ at time 40-305. All such task executions and actions are planned and calculated in a manner which reduces the total makespan. This schedule serves as the solution for the problem of multiple task executions by UAVs in indoor environment.

\subsection{Model description}

Based on the assumptions defined in Sect. 3.1, a mathematical model which is inspired by the notations addressed by Akturk and Yilmaz in [2] is developed as follows. Note that a recharge action is a specialization of a task in this model.

Sets

$N_{t} \quad$ set of tasks, $N_{t}=\{1,2,3, \ldots, N\}$, in which $\mathrm{N}$ is the number of tasks

$N_{r} \quad$ set of pre-assigned candidates of recharge action, $N_{r}=\{N+1, N+2, \ldots, N+H N\}$, in which $N$ is the number of tasks and $H$ is the number of recharge slots

$N_{q} \quad$ set of tasks and recharge actions whose start and/or end position is at $q$

\section{Indices}

$h, i$ index for a task and a recharge action$h, i \in N_{t} \cup N_{r}$

$t \quad$ a planning period- $t \in\{1,2,3, \ldots, T\}$, in which $T$ is the last period of the planning horizon
Fig. 3 Representation of a schedule (the solution of the problem)

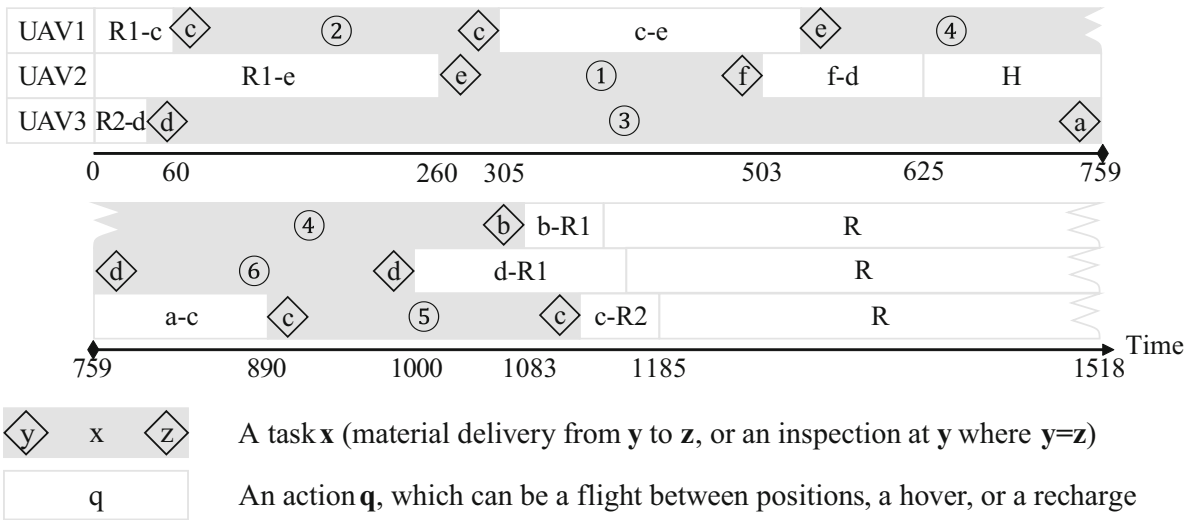


Table 4 Scheduled tasks and actions in Fig. 3

\begin{tabular}{llllrc}
\hline UAV ID & Task or action & Start position & End position & Start timestamp & End Timestamp \\
\hline 1 & R1-c & R1 & c & 0 & 60 \\
1 & 2 & c & c & 60 & 305 \\
1 & c-e & c & e & 305 & 533 \\
1 & 4 & e & b & 533 & 1083 \\
1 & b-R1 & b & R1 & 1083 & 1143 \\
1 & R & R1 & R1 & 1143 & 3843 \\
2 & R1-e & R1 & e & 0 & 260 \\
2 & 1 & e & f & 260 & 503 \\
2 & f-d & f & d & 503 & 625 \\
2 & H & d & d & 625 & 759 \\
2 & 6 & d & d & 759 & 1000 \\
2 & d-R1 & d & R1 & 1000 & 1160 \\
2 & R & R1 & R1 & 1160 & 3860 \\
3 & R2-d & R2 & d & 0 & 40 \\
3 & 3 & d & a & 40 & 759 \\
3 & a-c & a & c & 759 & 890 \\
3 & 5 & c & c & 890 & 1125 \\
3 & c-R2 & c & R2 & 1125 & 1185 \\
3 & R & R2 & R2 & 1185 & 3885 \\
\hline
\end{tabular}

$k \quad$ index for the order of a task executed by a UAV$k \in\{1,2,3, \ldots, K\}$, in which $\mathrm{K}$ is the total number of tasks executed by a UAV

$r \quad$ index for the order of a recharge action executed by a UAV $-r \in\{1,2, \ldots, R\}$, in which $\mathrm{R}$ is the latest index of recharge which is done by a UAV throughout the planning horizon, and the end time of the 0th recharge only indicates the beginning of the planning horizon

$u \quad$ index for a UAV- $u \in\{1,2,3, \ldots, U\}$, in which $U$ is the number of UAVs

$p, q$ index for a position- $p, q \in\{1,2,3, \ldots, Q\}$, in which $Q$ is the number of positions

\section{Decision variables}

$\begin{array}{ll}X_{i u k} & = \begin{cases}1 & \text { iftaskiisassignedasthek-thtaskofUAVu } \\ 0 & \text { otherwise }\end{cases} \\ Y_{h i} & = \begin{cases}1 & \text { iftaskhprecedestaskionthesameUAV } \\ 0 & \text { otherwise }\end{cases} \\ S_{i u k} & \in Z^{+}, \text {start time of task } i \text { assigned to UAV } u \text { as its } k \text { - } \\ F_{\text {iuk }} & \in Z^{+}, \text {finish time of task } i \text { assigned to UAV } u \text { as its } \\ Z_{\text {iukr }} & k \text {-th task } \\ & = \begin{cases}1 & \text { iftaskiisthek-thtaskandther-threchargeactionofUAVu } \\ 0 & \text { otherwise }\end{cases} \end{array}$

\section{Parameters}

$w_{i} \quad$ task execution time of task $i$
$P_{h i}=1$ if task $h$ precedes task $i$

$O_{i} \quad$ start position of task $i$

$D_{i} \quad$ end position of task $i$

$d_{p q} \quad$ distance between position $p$ and $q$

$v_{0} \quad$ fixed flight speed of UAV (with or without payload)

$C$ battery capacity - upper bound of the flight time between battery recharges

Tasks and recharge actions scheduled in the mathematical model consist of two disjoint sets. The first set called $N_{t}$ comprises the tasks defined in the task layer in Sect. 3.1. As mentioned above, three types of task covered in this study consists of single inspection, compound inspection, and material handling tasks. Another set called $N_{r}$ comprises the recharge actions. The total number of recharge actions can be the same as the tasks, in the worst case. Each recharge action can be executed in one of the recharge slots in multiple recharge stations. Note that a recharge station may consist of multiple recharge slots. In the mathematical model, each recharge slot has a unique position. Correspondingly, the number of the predefined recharge actions is the product of the number of tasks and the number of all recharge slots. Unlike tasks, recharge actions do not have precedence constraint. As mentioned in the model description, the battery level is conversed in time dimensional unit.

Decision variable $X_{i u k}, Y_{h i}, S_{i u k}, F_{i u k}$ follows the modelling formalism of Akturk and Yilmaz [2]. The extended model covers the battery constraint and collision avoidance constraint with additional decision variables $Z_{i u k r} . X_{i u k}$ and 
$Y_{h i}$ define the task sequence of each UAV. $S_{i u k}$ and $F_{i u k}$ define the start time and finish time of each task, respectively. $Z_{\text {iukr }}$ discriminates the recharge action from the task sequence. This leads to the distinction of the consecutive recharge actions, which is then used to calculate the battery consumption between recharges. The mathematical model formulation is given by the following objective function,

Minimize $\left(\max _{i, u, k} F_{i u k}\right) \quad \forall i \in N_{t} \cup N_{r}, \quad \forall u, k$

subject to

$$
\begin{aligned}
& \sum_{u=1}^{U} \sum_{k=1}^{K} X_{i u k}=1 \quad \forall i \in N_{t} \\
& \sum_{u=1}^{U} \sum_{k=1}^{K} X_{i u k} \leq 1 \quad \forall i \in N_{r} \\
& \sum_{i \in N_{t} \cup N_{r}} X_{i u k} \leq 1 \quad \forall u, k \\
& w_{i} X_{i u k} \leq F_{i u k}-S_{i u k} \quad \forall i \in N_{t} \cup N_{r}, \quad \forall u, k \\
& F_{h, u, k-1}+\frac{d_{D_{h}} O_{i}}{v_{0}} Y_{h i} \leq S_{i u k} \quad \forall h, i \in N_{t} \cup N_{r}, \quad \forall u, k \\
& X_{h, u, k-1}+X_{i u k}-1 \leq Y_{h i} \quad \forall h, i \in N_{t} \cup N_{r}, \quad \forall u, k \\
& \sum_{u=1}^{U} \sum_{i \in N_{t} \cup N_{r}} X_{i u k} \leq \sum_{u=1}^{U} \sum_{i \in N_{t} \cup N_{r}} X_{i, u, k-1} \quad \forall k \\
& F_{h v l} P_{h i} \leq S_{i u k} \quad \forall h, i \in N_{t}, \quad \forall q, u, v, k, l \\
& \left(S_{h v l}-F_{i u k}\right)\left(S_{i u k}-F_{h v l}\right) \leq 0 \quad \forall h, i \in N_{q}, \quad \forall q, u, v, k, l
\end{aligned}
$$

$\sum_{i \in N_{r}} \sum_{k=1}^{K} S_{i u k} Z_{i u k r}-\sum_{h \in N_{r}} \sum_{l=1}^{K} F_{h u l} Z_{h, u, l, r-1} \leq C \quad \forall u, r$

$\sum_{k=1}^{K} Z_{i u k r} \leq 1 \quad \forall i \in N_{r}, \quad \forall u, r$

$F_{i u k} \leq C\left(1+\sum_{r=1}^{R} \sum_{l=1}^{k} \sum_{h \in N_{r}} Z_{h u l r}\right) \quad \forall i \in N_{t} \cup N_{r}, \quad \forall u, k$

The objective of the mathematical model is to minimize the total makespan of the operations. Constraints (2)-(6) are featured in Akturk and Yilmaz [2]. Constraint (2) assigns a task to be executed once by one UAV. Note that constraint (2) is defined only for tasks, and constraint (3) is defined for recharge actions. Constraint (4) assures each time slot of each UAV to be assigned to at most one task or recharge action. Equation (5) defines the relation between start time and finish time. Constraints 6 and 7 describe the task sequence and the time relation between tasks.
Constraint (8) is provided for the strictness of the mathematical model. It prevents empty slots in the middle of the time slot sequence of each UAV, which causes the associated decision variables $Y_{h i}, S_{i u k}, F_{i u k}$, and $Z_{i u k r}$ undefined. Constraint (9) defines the precedence constraint, and constraint (10) defines the position occupation constraint in a nonlinear form. The constraint assures that any pair of two tasks cannot be executed simultaneously. Constraint (11) describes the battery constraint in a nonlinear form. The time gap between every consecutive recharge action is restricted to be less than the battery capacity. It enforces the necessary amount of recharge actions accordingly. Constraint (12) is an assignment constraint making the relation between the task sequence and the recharge sequence of each UAV. Constraint (13) is a bin-packinglike constraint [41] for each UAV to schedule at least a minimum required number of recharges-assuming that the UAVs are fully charged in the beginning of the planning horizon.

In regard to the considered constraints, the problem in this paper draws an input from parallel machine scheduling (PMS) problem [37]. Since multiple tasks can be executed by multiple available UAVs, the UAVs can be analogized to parallel machines. The proposed problem covers the one which is preliminarily defined in [32], which is investigated under the constraint satisfaction programming (CSP) modelling formalism. PMS problem is one of the most reported works in the scheduling literature [12]. Garey and Johnson [15] showed that PMS problems with the objective of minimizing the makespan are categorized as NP-hard.

One constraint of the addressed problem is that the tasks which are assigned to the same position cannot be executed simultaneously. Under this constraint, not only the UAVs, but also the positions should be considered as resources. Several researchers have studied various scheduling problems with different constraints. Kellerer and Strusevisch [20] showed that the two-machine problem with one additional resource type is NP-hard. Hou and Guo [19] proposed an MIP model of parallel machine scheduling with resource constraints and sequence-dependent setup times, which was also reported to be NP-hard. Another crucial constraint of the addressed problem is the capacitated battery constraint. It inflicts necessary UAV recharges which proportionally depend on the execution of the assigned tasks. From the classic scheduling problem, this issue has been discussed as a preventive maintenance problem. Qi et al. [35] proved that the maintenance scheduling on a single machine is NP-hard problem by reducing it to distance-constrained VRP (vehicle routing problem). $\mathrm{Xu}$ et al. [49] modelled parallel machine scheduling with $\epsilon$-almost periodic maintenance constraint, which means that the time between any two consecutive maintenance is within the interval $\left[T, T^{\prime}\right]$, where $T$ and $T^{\prime}$ 
are positive real numbers and $T^{\prime}-T<\epsilon$. Consequently, it can be concluded that the nature of the addressed problem in this paper is also NP-hard.

There are various possible objectives which can be considered in the UAV scheduling problem, as in various other scheduling problems. Among different objectives $[34,40]$, minimization of makespan is selected to be optimized in this paper. A makespan indicates the general throughput of the UAV scheduling system, and an optimized makespan means an efficient UAV schedule which maximizes this throughput where tasks are executed in a time-efficient manner. It can also be considered as a measure of the battery consumption and resource utilization. To achieve this objective, a proposed heuristic is incorporated with particle swarm optimization (PSO) algorithm which will be discussed in the following section.

\section{Application of PSO for UAV scheduling system}

Characteristics of the presented problem distinguish its nature as NP-hard. Approaches based on branch \& bound and branch \& cut are tedious in terms of computation time. A promising alternative to those methods is a metaheuristic algorithm. Metaheuristics use different concepts derived from artificial intelligence and evolutionary algorithms, which are inspired from mechanisms of natural evolution [40]. From the literature, it could be found that metaheuristics can also be called as soft computing techniques, evolutionary algorithms, and nature-inspired algorithms. Metaheuristic methods are designed for solving a wide range of hard optimization problems without having to adapt deeply into each problem. These algorithms are fast and easy to implement [44]. From the literature review, it could be seen that different metaheuristic algorithms have been proposed to obtain feasible solutions for general scheduling problems [16].
Particle swarm optimization (PSO) algorithm is a swarm-based stochastic optimization technique developed by Kennedy and Eberhart [21] based on the characteristics of social behaviour of birds in flocks or fish in schools. This metaheuristic algorithm is chosen to be incorporated in the proposed methodology in this paper. PSO algorithm does not involve the usage of genetic operators (mutation and crossover) which are commonly used for evolutionary algorithms. On top of that, PSO utilizes a minimum number of parameters to achieve the near-optimum solution in a faster computation time [31]. These factors have influenced the authors to choose PSO to solve the presented problem, where there is a requirement of generating a fast and feasible schedule in a real-time application.

PSO is an optimization method based on the population which is referred as swarm in this paper. Simplicity in application, easy implementation, and faster convergence of PSO have made the algorithm widely acceptable among researchers for solving different types of optimization problem [18]. Different variants of PSO have been developed and employed by researchers to solve scheduling problems. This paper employs the standard PSO [21] model to solve the UAV scheduling problem. The pseudocode of PSO is presented in Algorithm 1. All particles in the swarm share the global best particle information which helps to search towards the best position in the search space. Each single solution in the search space is called as a particle. All particles are to be evaluated by an objective function (explained in the following section) which is to be optimized. Each particle in the swarm searches for the best position, and it travels in the search space with a certain velocity. Best fitness encountered by each particle (local best) is stored and the information is shared with other particles to obtain the best particle (global best). In this paper, the velocity and position update of PSO are based on [36] to suit the presented UAV scheduling problem.

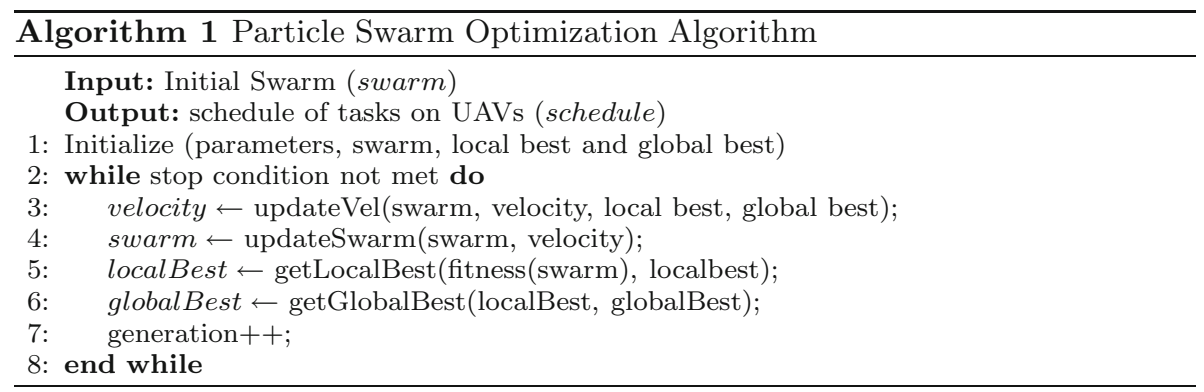


Table 5 Example data and parameters for PSO procedure

\begin{tabular}{lll}
\hline Data or parameter & Notation & Value \\
\hline Local best particle & ${ }^{l o} P_{i}^{t}$ & {$[1,2,4,6,5,8,3,7,10,9,11,12]$} \\
Global best particle & $G^{t}$ & {$[2,6,1,4,3,5,7,8,10,9,11,12]$} \\
Particle & $P_{i}^{t}$ & {$[1,2,4,6,5,8,7,3,10,9,12,11]$} \\
Initial velocity & $v_{i}^{t}$ & $(6,7),(10,11)$ \\
Learning coefficient 1 & $c_{1}$ & 1 \\
Learning coefficient 2 & $c_{2}$ & 2 \\
Velocity coefficient & $U_{1}$ & 0.2 \\
Velocity coefficient & $U_{2}$ & 0.4 \\
\hline
\end{tabular}

The procedural steps of the PSO algorithm are given below:

Step 1 The initial population is generated based on priority rules. Initial velocities for each particle are randomly generated.

Step 2 Based on the objective function, each particle is evaluated.

Step 3 Each particle remembers the best result achieved so far (local best) and exchange information with other particles to obtain the best particle (global best) among the swarm.

Step 4 The velocity of the particle is updated using Eq. (14), and the position of the particle is updated using Eq. (15).

Velocity update equation:

$v_{i}^{t+1}=v_{i}^{t}+\underbrace{c_{1} *\left[U_{1} *\left({ }^{l o} P_{i}^{t}-P_{i}^{t}\right)\right]}$ cognitivepart $+\underbrace{c_{2} *\left[U_{2} *\left(G^{t}-P_{i}^{t}\right)\right]}$ socialpart

where $U_{1}$ and $U_{2}$ are known as velocity coefficients (random numbers between 0 and 1), $c_{1}$ and $c_{2}$ are known as learning (or acceleration) coefficients, $v_{i}^{t}$ is the initial velocity (which comprises pairs of transposition-see Sect. 4.1.2), ${ }^{l o} P_{i}^{t}$ is the local best, $G^{t}$ is the global best solution at generation $t$, and $P_{i}^{t}$ is the current particle position.

Acceleration coefficients $c_{1}$ and $c_{2}$ are the important parameters of PSO. Parameter $c_{1}$ is used to guide the particle towards its own (local) best position, and this behaviour enhances the grouping ability and keeps the diversity of the population. Parameter $c_{2}$ acts as a convergence factor that attracts all the particles towards the global best position.

To suit the considered problem, the particle is represented in the form of a task sequence (for an example, refer the value of particle in Table 5). This enables a tractable update during the numerous iterations. A particle represents a feasible solution which consists of a sequence of tasks. The sequence shall satisfy the precedence relationships, and it is initially generated based on a priority rule. The priority rules which are considered in this study are explained in Sect. 4.1.1. A velocity of a particle is a collection of transpositions, where each transposition is represented as position indices of two tasks in a sequence (particle) which will be swapped accordingly (during the velocity update). In this study, the position index starts from 0 instead of 1 for a seamless implementation (from the programming perspective).

Each particle changes its position according to its velocity, which is randomly generated towards the local best $\left({ }^{l o} P_{i}^{t}\right)$ and the global best $\left(G^{t}\right)$ positions. The moving direction of the particle is decided by three parts as shown in Eq. (14). These three parts comprise the initial velocity $v_{i}^{t}$ of the particle at a particular iteration, the optimum distance of ${ }^{l o} P_{i}^{t}-P_{i}^{t}$ that the particle passed (this is known as a cognitive part which controls the exploration of the particle based on its own exploration experience), and the optimum distance of $G^{t}-P_{i}^{t}$ that the particle swarm passed (this is known as a social part which helps in collaborating with other particles based on the group exploration experience [7]). The proportions of impact for cognitive and social parts are decided based on the coefficients $c_{1}$ and $c_{2}$. These coefficients determine whether a particle prefers to move closer to the local best or global best position.

A particle moves from its current position to the new position using Eq. (15). The position of every particle in the swarm is updated in every iteration.

Position update equation:

$P_{i}^{t+1}=P_{i}^{t}+v_{i}^{t+1}$

Different operators used in computing the particle velocity and position are explained as follows [36].

- Subtraction (position-position) operator Let two positions $x_{1}$ and $x_{2}$ represent two different task sequences (see Sect. 4.1.1). The difference of $x_{2}-x_{1}$ is a velocity $v$. In Eq. (14), subtracting two positions, i.e. ${ }^{l o} P_{i}^{t}-P_{i}^{t}$ results in a velocity which is a set of transpositions (see Sect. 4.1.2). The difference of the positions of two particles can be obtained in various implementation ways. For instance, each element 
(A) in the first particle is compared with element (B) in the second particle at the same position index. When they are different, the position of B is searched in the first particle. That position index is then coupled with the position index of A. After completing all iterations, a collection of position index pairs (for performing a transposition) is obtained. This procedure has been widely used in $[31,36]$.

- Addition (position + velocity) operator Let $x_{1}$ be a position, and $v$ be a velocity of the particle. New position $x_{2}$ is calculated by applying each transposition pair in $v$ consecutively to $x_{1}$.

- Addition (velocity + velocity) operator Let $v_{1}$ and $v_{2}$ be two velocities (which may come from either one of the three parts of Eq. (14)). In order to calculate $v_{1}+v_{2}$, the list of transpositions combines the subsets of transpositions from $v_{1}$ and $v_{2}$.

- Multiplication (constant* velocity) operator Let $c$ be a constant and $v$ be a velocity (which consists of one or more transposition pairs). The operation of $c * v$ results in a new velocity with an equal or less number of transposition pairs, where the first $c * 100 \%$ proportion of the collection is selected.

Step 5 Go back to step 2 until the termination criterion is met. Equations (14) and (15) describe the path in which the particles move in the search space.

In this paper, PSO is used to optimize the proposed problem, where one of the key challenges encountered is the encoding of the particle. In most applications of PSO, particle position $P_{i}^{t}$ is directly denoted as the solution in the form of a sequence of tasks. The arrangement of the sequence is ensured to satisfy the precedence relationships. To illustrate how the velocity and position update work, an example is explained as follows-the notations of the required data or parameters are depicted in Table 5.

The current velocity of a particle is updated based on Equation (14) as follows.

$$
\begin{aligned}
v_{i}^{t+1} & =(6,7)(10,11)+0.2 * \\
& {[(1,2,4,6,5,8,3,7,10,9,11,12)} \\
& -(1,2,4,6,5,8,7,3,10,9,12,11)] \\
& +0.8 *[(2,6,1,4,3,5,7,8,10,9,11,12) \\
& -(1,2,4,6,5,8,7,3,10,9,12,11)] \\
& =(6,7)(10,11)+0.2 *(6,7)(10,11) \\
& +0.8 *(0,1)(1,3)(2,3)(4,7)(5,7)(10,11) \\
= & (6,7)(10,11)(0,1)(1,3)(2,3)(4,7)(5,7)
\end{aligned}
$$

Using Eq. (15), the current particle is updated to a new particle using the new velocity.

$$
\begin{aligned}
P_{i}^{t+1} & =(1,2,4,6,5,8,7,3,10,9,12,11) \\
& +(6,7)(10,11)(0,1)(1,3)(2,3)(4,7)(5,7) \\
& =(2,6,1,4,7,5,3,8,10,9,11,12)
\end{aligned}
$$

The products of coefficient values $c_{1}, U_{1}$ and $c_{2}, U_{2}$ represent the proportion which decides how many pairs of transposition would be copied to form the updated velocity. For example, when the proportion is $80 \%$ $\left(c_{2} * U_{2}=2 * 0.4=0.8\right), 80 \%$ of the pairs would be copied to the updated velocity. In this example, 6 pairs are formed when transpositions take place between the global best and the current particle. Based on this proportion, 5 pairs are chosen out of 6 for the updated velocity. However, if any of the pairs is already present from other transpositions or initial velocity, it is discarded. A repair mechanism is incorporated to convert an infeasible sequence to a feasible one which meets the precedence relationships-presented as Algorithm 3 in this paper.

\subsection{PSO entities}

To illustrate the explanation of different entities in PSO, a sample dataset presented in Tables 2 and 3 is used.

\subsubsection{Initial population}

Metaheuristic algorithms start with a random search state which iteratively evolves to find a near-optimum solution [34]. Despite the search state being random, the generation of the initial population is not purely random but guided by the priority (heuristic) rules. The purpose for doing this is to start the search from hypothetically good starting points rather than random ones, so that global optimum is more likely achieved in less computation time. In this paper, six priority rules (maximum rank positional weight, minimum inverse positional weight, minimum total number of predecessor tasks, maximum total number of follower tasks, maximum and minimum task time) presented in [34] are used to generate the initial particles. Two more priority rules based on the number of predecessor and follower tasks are added to the existing rules, and these are named as cumulative number of predecessor and follower tasks. Based on these eight priority rules, eight initial particles can be generated respectively. In regard to the number of initial particles, it is reported in the literature that a high initial population size improves the quality of the solution. Hence, up to forty initial particles are generated in the experiments. Each remaining particle apart from the aforementioned eight is generated by randomly swapping the tasks in one of the eight particles without violating the precedence relationships and produces a new one. Table 6 reports the set of initial particles generated using the 
Table 6 Priority rules for initial swarm generation

\begin{tabular}{llllllllllllll}
\hline Priority rules & \multicolumn{1}{c}{ Task sequence } \\
\hline Maximum ranked positional weight & 1 & 2 & 4 & 6 & 5 & 8 & 3 & 7 & 10 & 9 & 11 & 12 \\
Minimum inverse positional weight & 1 & 2 & 3 & 4 & 5 & 6 & 7 & 12 & 9 & 8 & 10 & 11 \\
Minimum total number of predecessors tasks & 1 & 2 & 3 & 4 & 5 & 6 & 7 & 9 & 8 & 10 & 11 & 12 \\
Maximum total number of follower tasks & 2 & 6 & 1 & 4 & 3 & 5 & 7 & 8 & 10 & 9 & 11 & 12 \\
Maximum task execution time & 3 & 2 & 1 & 4 & 7 & 9 & 6 & 12 & 5 & 8 & 10 & 11 \\
Minimum task execution time & 1 & 2 & 5 & 6 & 4 & 8 & 10 & 11 & 7 & 9 & 3 & 12 \\
Minimum number of cumulative predecessor & 1 & 2 & 3 & 4 & 5 & 6 & 7 & 9 & 12 & 8 & 10 & 11 \\
$\quad$ & & & & & & & & & & & & \\
Masks & 1 & 2 & 4 & 5 & 6 & 8 & 3 & 7 & 10 & 9 & 11 & 12 \\
\hline
\end{tabular}

priority rules and conformed with Algorithm 3 to satisfy the precedence relationships. The precedence relationships of tasks are available in Table 2. Each particle is structured as a string of tasks which are to be executed in the UAV operation.

\subsubsection{Initial velocity}

Each particle is assigned with velocity pairs, and these pairs are randomly generated. Based on the pilot experiments, it is decided to have different sizes of velocity pairs for different problems based on the number of tasks. Table 7 presents the maximum number of velocity pairs used in this research for different numbers of task. The velocity is updated from the second iteration using Eq. (14). The number of pairs is the same throughout all iterations. For example, if the number of tasks is within the range of $0-20$, the number of velocity pairs is selected as 2 .

\subsection{Schedule creation and evaluation}

In the addressed methodology, the initial population is generated according to the priority rules explained in

Table 7 Number of initial velocity pairs in regard to the number of tasks

\begin{tabular}{lc}
\hline Number of tasks & Maximum number of velocity pairs \\
\hline $0-20$ & 2 \\
$20-50$ & 10 \\
$50-100$ & 30 \\
\hline
\end{tabular}

Table 6. Tasks in the task sequence are scheduled, where each of them is assigned to a UAV to be executed in a particular timespan, one by one (per step) according to its order in the sequence. Figure 4 depicts the sequential steps of creating a schedule from a sequence of 12 tasks.

The aforementioned heuristic for creating a schedule from a task sequence is depicted in Algorithm 2. The idea behind this algorithm is to create a schedule which is driven to utilize the available resources in the following manners, which generally lead to a time-efficient characteristic:

- balanced

- task assignment towards the earliest available UAV (which indicates its relative idleness compared to other UAVs)

- safe

- no multiple UAVs are allowed to occupy a position simultaneously

- task execution follows the precedence relationship

- early

- recharge station which eventually delivers a recharged UAV to its next destination early is chosen

- the shortest makespan found during the search is selected as the final solution

The detailed procedure of the heuristic is explained in Algorithm 2. 


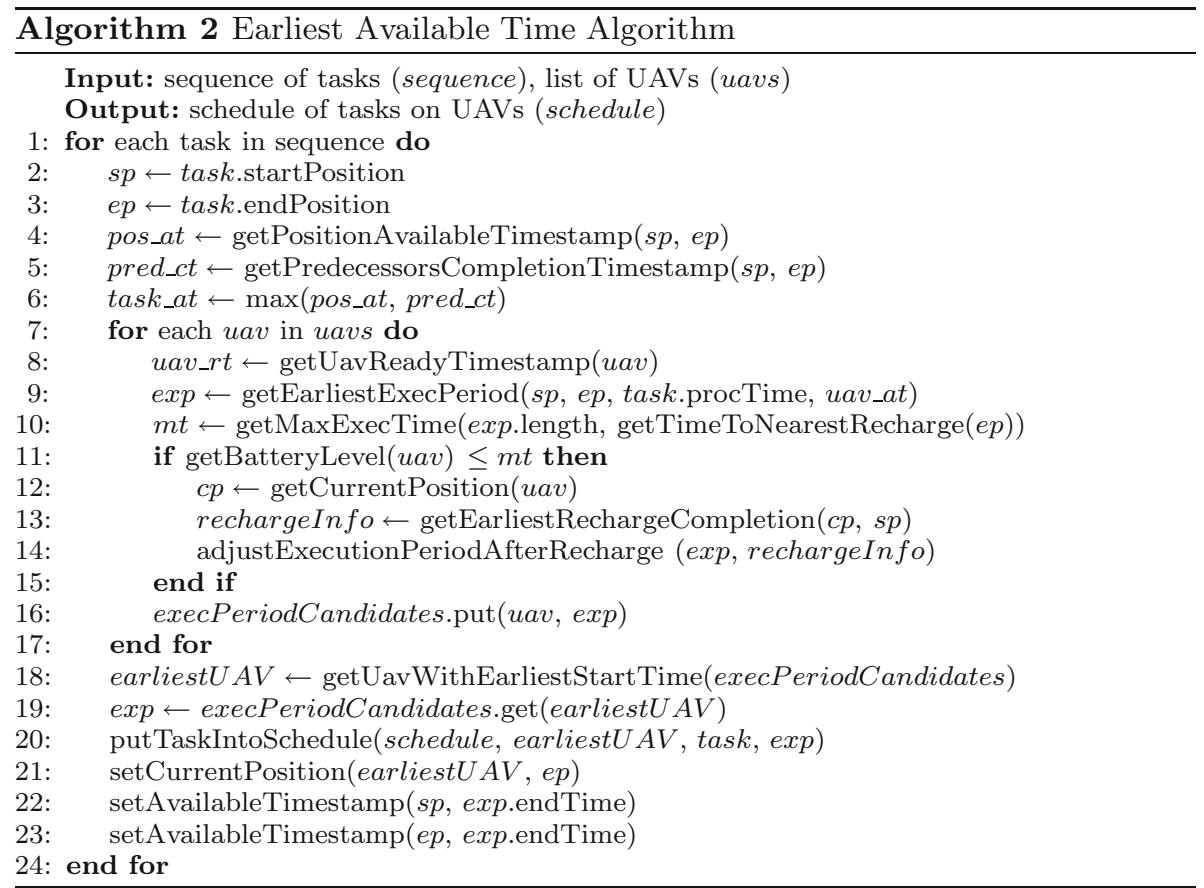

the fitness of the schedule is evaluated based on its

\begin{tabular}{|c|c|c|c|c|c|c|c|c|c|c|c|}
\hline step1 & step2 & step3 & step4 & step5 & step6 & step 7 & step8 & step9 & step10 & step11 & step 12 \\
\hline 3 & 2 & 1 & 4 & 6 & 5 & 7 & 9 & 12 & 8 & 10 & 11 \\
\hline
\end{tabular}

Fig. 4 Task scheduling steps

In the incorporated PSO, a particle indicates a solution (a schedule) which is selected through an iterative search process based on its fitness value. However, in regard to the position update process in PSO algorithm, it is difficult to define a step (of position update) due to the rigid structure of the schedule. A schedule may contain the following possible elements: flight, hover, wait-on-ground, recharge, and task. When a schedule is observed, there is a precise timespan in the schedule which most likely fits to only one particular task. An action's existence may also depend on another one (i.e. flight, hover, wait-on-ground, and recharge existence is relative to the task execution manner). Thus, when some elements in a schedule are swapped (manipulated to produce different solution), an infeasible schedule is frequently formed.

Considering the aforementioned conditions, the sequence representation (before it is transformed into a schedule) of the tasks is considered. Swapping tasks in a sequence forms a new one-it is tractable and robust. Since each task sequence uniquely corresponds to a schedule, it is valid to use task sequence as the particle representation. Obviously, the representation gap (of sequence and schedule) is filled with the proposed heuristic method, which creates a schedule from a task sequence. In the end, makespan. In this manner, both position update operation (in PSO iteration) and solution fitness evaluation are done seamlessly.

According to Algorithm 2, each task in the sequence (line 1) is put into the schedule, based on the earliest available time characteristic of the involved objects, described as follows.

1. Task availability check

(a) Position availability (line 2)

An abstraction of task is broken down into possible flights and other actions (e.g. pickup and release payload, inspection/capture image), which involves start position and end position. The time spent at start and end position is not defined for any task in this study. Hence, both positions are occupied during the whole execution time of the respective task. Consequently, start position and end position are checked for its occupancy status when a task is picked to be put into the schedule. The latest released (not occupied) timestamp is used for the position availability timestamp.

(b) Task precedence (line 3)

A task is checked for its existing preceding task. If preceding tasks exist, the last completion timestamp of them will be set as the earliest available timestamp for the task. If there is no preceding task, then the task is available at time 0 . 
2. UAV availability check

(a) UAV ready time (8) Task occupancy of each UAV is checked. The moment it goes to idle after completing the most recent task is recorded as its ready time.

(b) Battery level (lines 10-11)

After a task execution, a UAV must have enough battery level to at least fly towards the nearest recharge station.

(c) Recharge time (lines 10-15)

The battery check which is done in the part of line 10 is done to guarantee a sufficient battery level to go to at least the nearest recharge station. If a UAV doesn't have enough battery to go to the recharge station after executing a task, then it needs to go to the recharge station with the earliest recharge completion time before flying to the start position of the task and execute it. To ensure that the UAV is fully charged (at a capacity of $1200 \mathrm{~s}$ flight), an actual recharge timespan is always set to $2700 \mathrm{~s}$; it is the time required to do a full battery recharge. Recharge time is the summation of round-trip time and actual recharging timespan (which may be longer than $2700 \mathrm{~s}$ due to the delayed recharge station availability time).

(d) Recharge station availability (line 13)

A limited number of recharge slots at the recharge station are considered. When all recharge slots at a recharge station are occupied, its earliest available time is the earliest timestamp when an occupying UAV leaves the recharge station. The next selection criteria is based on the shortest round-trip (end position of previous task $\rightarrow$ recharge station $\rightarrow$ start position of current task) time to a recharge station. It means that the nearest recharge station might not be preferred due to its far distance to the start position of the current task.

3. Overall availability check (lines 4-18)

Incorporating the aforementioned task availability and UAV availability check, the start timestamp of the respective task is calculated. A UAV with the earliest overall available time is selected (line 18), and the task is put into the respective UAV's schedule. Completion (end) timestamp of a task potentially performed by each UAV is also included in the execution period calculation (line 9) and used to release the occupied positions (lines 22-23).

For readability, putTaskIntoSchedule (line 24) in Algorithm 2 encapsulates the following processes:

1. Insert a task into the schedule of the selected (earliest available) UAV

2. Insert a recharge action (if required) into the schedule of the selected UAV

(a) update occupancy status of the respective recharge slot

3. Update battery level of the respective UAV

4. Insert hover and wait-on-ground in between tasks in the schedule when needed

These supplementary actions are required to generate a feasible schedule of UAV operations [22].

To illustrate the usage of the heuristic described in Algorithm 2, steps $1-7$ of the task scheduling process for a given task sequence in Fig. 4 are presented. Steps 1 and 2 are depicted in Figs. 5 and 6, while steps 3-7 are presented in "Appendix A". Steps 8-12 which are principally doing the same procedure as the previous ones are not presented in the paper. In accordance with the explanation of Algorithm 2, task and UAV availability check are performed every time a task is picked to be put into the schedule. Detailed stepwise procedure for the first two steps is explained below the figures. Task, UAV, and overall availability check are referred as point (a), (b), and (c), respectively.

After completing all 12 steps, a schedule of 12 task executions is obtained. The schedule has a makespan of $4963 \mathrm{~s}$, which is called as fitness value of the particle (in the incorporated PSO).

Furthermore, when there is an infeasible sequence (which doesn't meet the precedence relationships) during the position update, a repair mechanism is conducted based on Algorithm 3. 


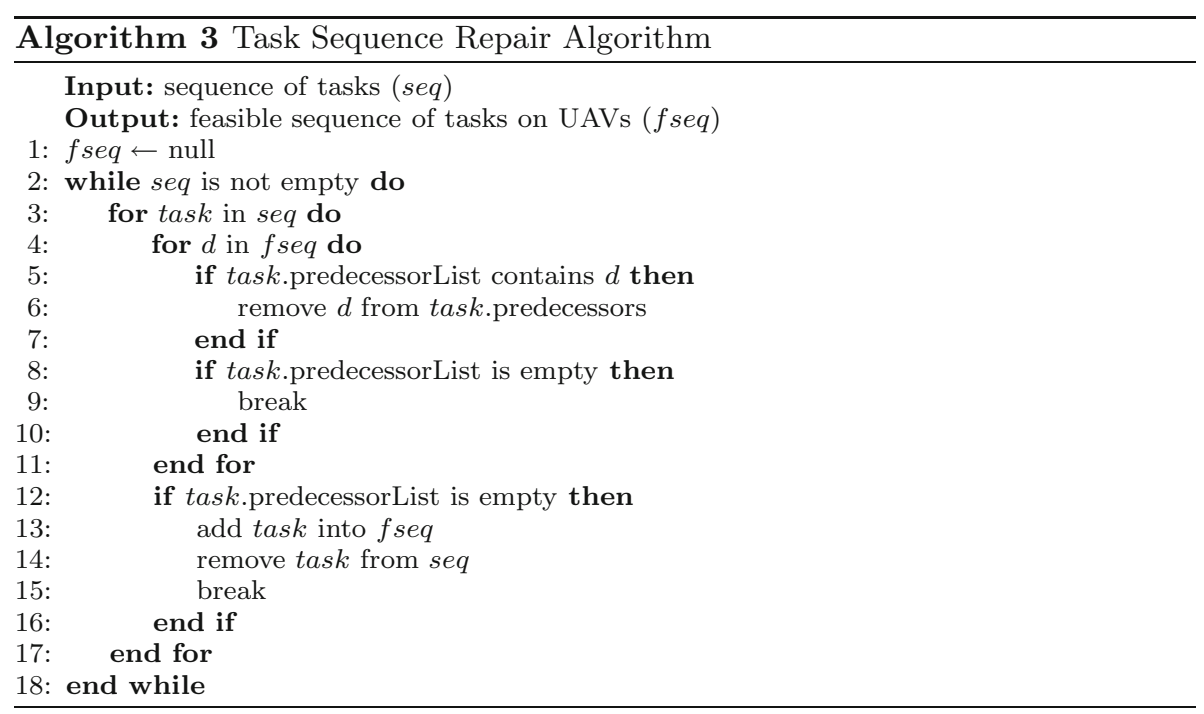

Fig. 5 Output of step 1 of the schedule creation heuristic

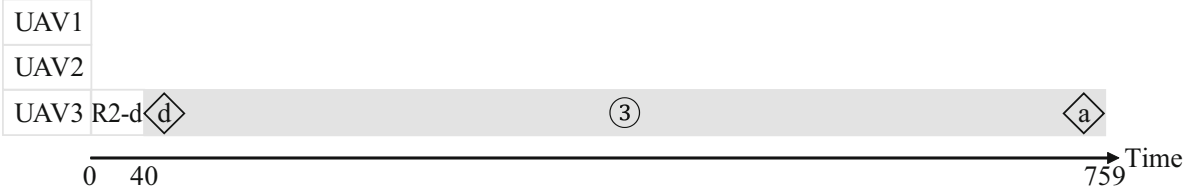

The process can be pictured as moving the tasks from a sequence to a new one where precedence feasibility is held along the way. An agent visits the tasks in the task sequence sequentially as long as there is at least one task in the original sequence (lines 1-2). It checks each predecessor in the (immediate) predecessor list of the currently visited task. If it is contained in the new sequence, then it shall be removed from the list (lines 3-10). If the list of predecessors of the currently visited task is empty, then this task is ready to be moved into the new sequence (lines 11-15) - the task is added at the end of the sequence (line 12). Otherwise, it searches the following tasks (according to the sequence) to find a task which is ready to be moved (line 2). In the end, a feasible sequence that meets the precedence relationships is produced, which represents one valid particle in the PSO framework.

The sample data (Tables 2 and 3) being used in this section bring an example of task dataset which strongly displays the logic of Algorithm 2. For further analysis and evaluation, several datasets with different data volumes are generated. Respectively, the mechanism of generating the data is also presented in the following section.

\section{Numerical experiments}

To examine the behaviour and the performance of the proposed methodology, numerical experiments are conducted based on 3 task datasets. Several different treatments are given during those experiments and explained in detail in Sects. 5.2.1 and 5.2.2. The proposed algorithm is coded on a Java platform, and the numerical experiments are conducted on an Intel Core i7 processor $(2.9 \mathrm{GHz})$ with $32 \mathrm{~GB}$ of RAM.

\subsection{Data generation}

Task dataset used in the experiment is generated based on a test flight conducted in the laboratory. It is conducted to measure the speed of the UAV movement in a real-world indoor environment. Based on the required test case described in task layer (see Sect. 3.1), several types of task are classified in Table 8. A single inspection task captures an observation image of a certain area of interest; its processing time is $20-80 \mathrm{~s}$. A compound inspection task captures multiple observation images of several areas of interest; its processing time is 100-200 s. Unlike a single inspection task, compound inspection task might contain flight actions between captures. Without such an abstraction, the level of detail will cause a high number of steps in a solution, which obviously affects the computation time in finding one. The next type of task is material handling. Material handling consists of pickup, transport flight, and release. Its processing time is $30 \mathrm{~s}$ for each pickup and release $(30+30=60 \mathrm{~s})$, while transport flight varies according to the origin and destination positions.

During the scheduling process, a task has five attributes attached: task identifier, origin position, destination 
Fig. 6 Output of step 2 of the schedule creation heuristic

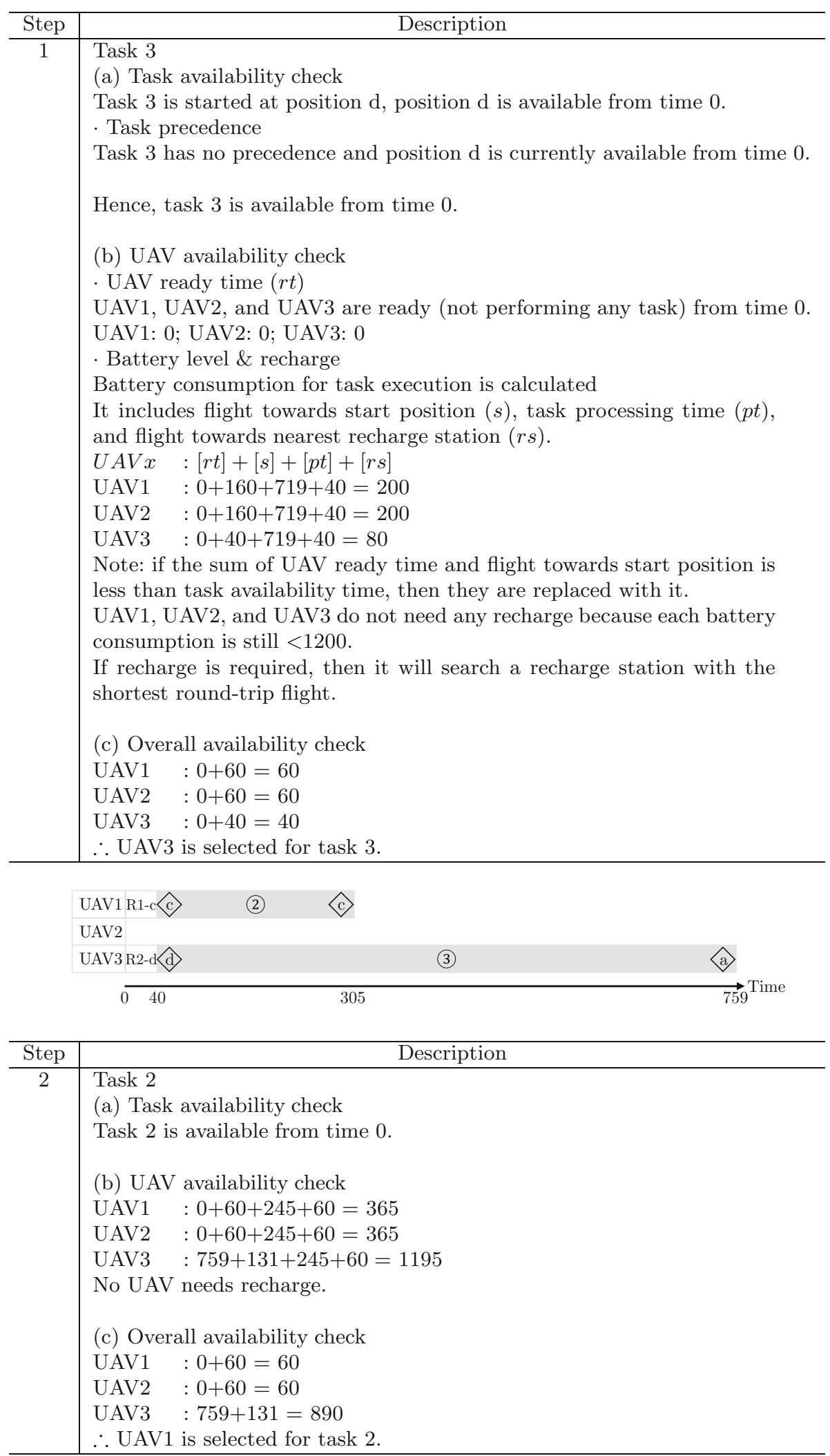


Table 8 Task types

\begin{tabular}{ll}
\hline Task type & Processing time (s) \\
\hline Single inspection & $20-80$ \\
Compound inspection & $100-200$ \\
Material handling & $60+$ flight_time \\
\hline
\end{tabular}

Table 9 Task attributes

\begin{tabular}{ll}
\hline Attribute & Data type \\
\hline Task identifier & Integer \\
Origin position & String \\
Destination position & String \\
Processing time & Integer \\
Predecessor list & Integers \\
\hline
\end{tabular}

position, processing time, and predecessor list (see Table 9). Task identifier is represented as a unique integer value, which means that no multiple tasks share the same value. Origin position and destination position are represented as a unique string each, which acts as a position name and a position identifier simultaneously. Processing time is represented as an integer value where its execution time shall never exceed the battery capacity of the UAV (Eq. (16)). Predecessor list is represented as a set of integers which indicates the identifiers of the preceding tasks.

execution_time $=$ preparation_flight + processing_time

$$
\text { + towards_recharge_flight }
$$

execution_time $\leq$ UAV_BATTERY_CAPACITY

\subsection{Parameter analysis and performance evaluation}

In the proposed method, the granularity of the processed data can be seen as a sequence. From a particular sequence, a schedule is created using a heuristic based on the proposed earliest available time algorithm. The schedule is then evaluated through its makespan (the total time required for completing all tasks based on a schedule). The process is done iteratively and executed in a manner according to the PSO algorithm. To control the performance of this PSO algorithm, i.e. tendency of optimality level and convergence speed, a set of parameters need to be configured. Through the conducted experiments, the respective performance of each set of parameters is measured and evaluated in Sect. 5.2.2 to decide the default

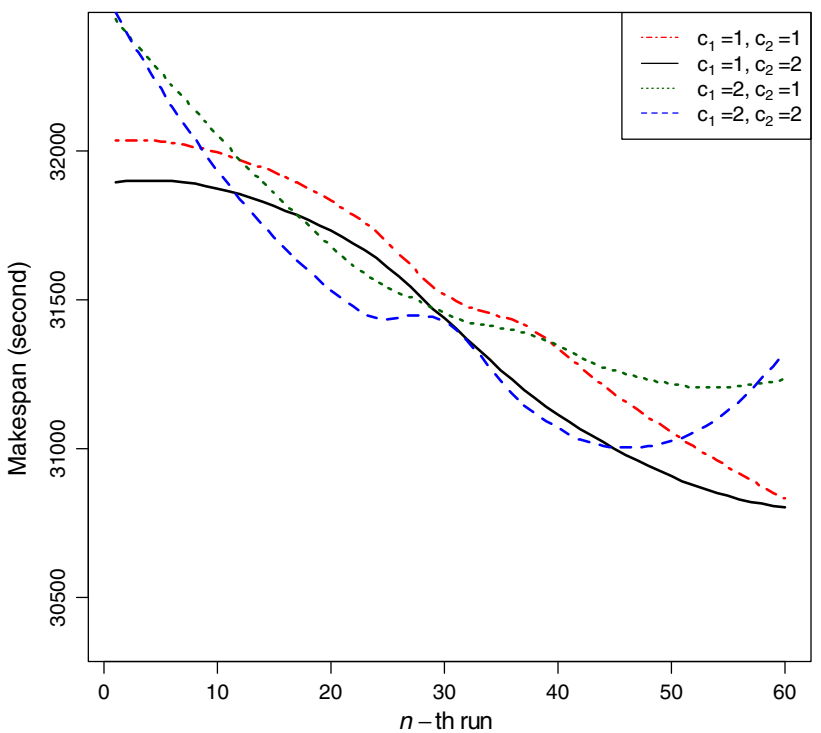

Fig. 7 Overall makespans on 100 tasks

parameter values which most likely bring the best result in regard to the task datasets.

In Sect. 5.2.1, the following parameters are analysed:

1. Size of initial population

Initial particles in the initial population serve as the initial starting points of the search. The more varying the starting points are, spread at different locations throughout the search space, the better chance the search has in reaching the global optimum instead of getting trapped at a local one.

2. Number of pairs in initial velocity

To cover a sufficient exploration area of the respective solution search space, one must adjust the number of pairs along with the escalation of the number of tasks. By doing so, a particle is capable to explore its surrounding search space and more likely to find a local best particle in that area. In the other way around, a lower number of pairs in initial velocity minimize the local exploration and force the search to rely more on the variety of particles in the initial swarm alone.

3. Value of learning coefficients $\left(c_{1}\right.$ and $\left.c_{2}\right)$ and velocity coefficients $\left(U_{1}\right.$ and $\left.U_{2}\right)$

According to Eq. (14) for velocity update, $U_{1}$ and $U_{2}$ are fraction numbers randomly generated ranging from 0.0 to 1.0. Furthermore, because of $c_{1}$ and $c_{2}$ multiplied, respectively, with $U_{1}$ and $U_{2}$, they will control the search direction. $c_{1} U_{1}$ and $c_{2} U_{2}$ will decide the number of pairs used from the distance of the current particle towards the local and global best particle, respectively. Since constant $c_{1}$ is set to be smaller than constant $c_{2}$, there will be a tendency of getting more pairs produced from the social part-from the global 
Table 10 Trade-off relationships among PSO parameters

\begin{tabular}{|c|c|c|}
\hline \multirow[t]{2}{*}{ Param. } & \multicolumn{2}{|l|}{ Treatment effect } \\
\hline & Increased & Decreased \\
\hline$c_{1}$ & $\begin{array}{l}\text { More pairs are derived from local best schedule sequence; it allows } \\
\text { the search to step over a local optima and possibly find a global } \\
\text { optimum schedule }\end{array}$ & $\begin{array}{l}\text { Less pairs are derived from local best schedule sequence; it } \\
\text { discourages exploration towards various directions in the } \\
\text { solution space }\end{array}$ \\
\hline$c_{2}$ & $\begin{array}{l}\text { More pairs are derived from global best schedule sequence; it drives } \\
\text { the search quickly towards the overall best schedule so far }\end{array}$ & $\begin{array}{l}\text { Less pairs are derived from the global best schedule; it } \\
\text { slows down the convergence speed and allows each } \\
\text { particle to have more room for exploration }\end{array}$ \\
\hline$U_{1}$ & Enhance the behaviour of increased/decreased $c_{1}$ & \\
\hline$U_{2}$ & Enhance the behaviour of increased/decreased $c_{2}$ & \\
\hline $\begin{array}{l}\text { Number of } \\
\text { initial } \\
\text { particles }\end{array}$ & $\begin{array}{l}\text { More starting points are spread out in the solution space, which } \\
\text { reduce the possibility to get trapped at a local optima, while } \\
\text { promoting a high-quality feasible solution in a quick time }\end{array}$ & $\begin{array}{l}\text { Less scattered starting points are established and less } \\
\text { pulling-each-other-out among particles; it promotes a } \\
\text { very quick convergence to the search }\end{array}$ \\
\hline
\end{tabular}

best sequence. Consequently, all particles in the swarm are alerted and encouraged to move towards the global best particle, while being less encouraged to move towards its own self-obtained local best particle. The movement of all particles towards the global best particle indicates the action of convergence during the whole search process, while the movement of each particle towards its local best particle allows the swarm to still explore towards various other directions to get potentially better global best particle.

\subsubsection{Parameter analysis}

Three different task datasets are used in the experiment, each consists of 12, 50 and 100 tasks, operated by 3 UAVs. In the experiments, makespans of the generated schedules based on various combinations of parameters: $c_{1}, c_{2}$, and initial population size on 3 different task datasets are measured. Further details can be seen in Fig. 13 in Appendix, whose summary is representatively depicted in Fig. 7. Systematically, the granularity of the experimental run is explained as follows.

1. There are 4 combinations of $c_{1}$ and $c_{2}$ treatment. Both $c_{1}$ and $c_{2}$ are set to 1 and 2 in all possible different scenarios. This treatment is considered to still give a room of exploration while converging more towards the suspected global optimum point during the search. For instance, when the value of $c_{1}$ is set to 2 , the update tendency of the velocity towards the local best is doubled (compared to the one with $c_{1}=1$ ) enabling the particles to be less explorative.

2. Each combination of $c_{1}$ and $c_{2}$ is applied on 3 task datasets: 10, 50, and 100 tasks.

3. Each task dataset is treated with either one out of 3 different sizes of initial population: 8,20 , and 40 particles Three different sizes of initial population are used in the presented scenarios. Eight initial particles are used in regard to the formulated priority rules which define the initial start points in a heuristic manner. Afterwards, the size is increased to 20 and 40, which are generated based on those 8 particles, to spread out the coverage of the initial search statewith an expectation to explore towards the direction of the global optimum solution. The initial population size is not set any higher to prevent a too dominant role of the generated initial particles and to allow the search to show its learning behaviour to be used in the parameter analysis.

4. Each treatment of a particular initial population size is run for 20 times.

Consequently, there are: $20 * 3 * 3 * 4=720$ runs (and 720 makespans, respectively) in total. Furthermore, a detailed observation about the treatments is presented in Table 10 .

From the depicted results in Fig. 13, several characteristics can be drawn as follows.

1. Makespan decreases as the size of population increases.

We have three treatments for the initial population size: 8,20 , and 40 . The first treatment is 8 initial particles because there are 8 priority rules for generating an initial swarm in Table 6, where each rule produces one particle. This initial population size is then doubled to make a significant difference with the previous treatment; it is roughly rounded up to 20 . Afterwards, the initial population size is doubled again to 40 . From those 3 treatments, one can find that as the initial population size increases from 8,20 to 40 , the makespan of the obtained solution decreases. This characteristic tends to hold true for all combinations of 


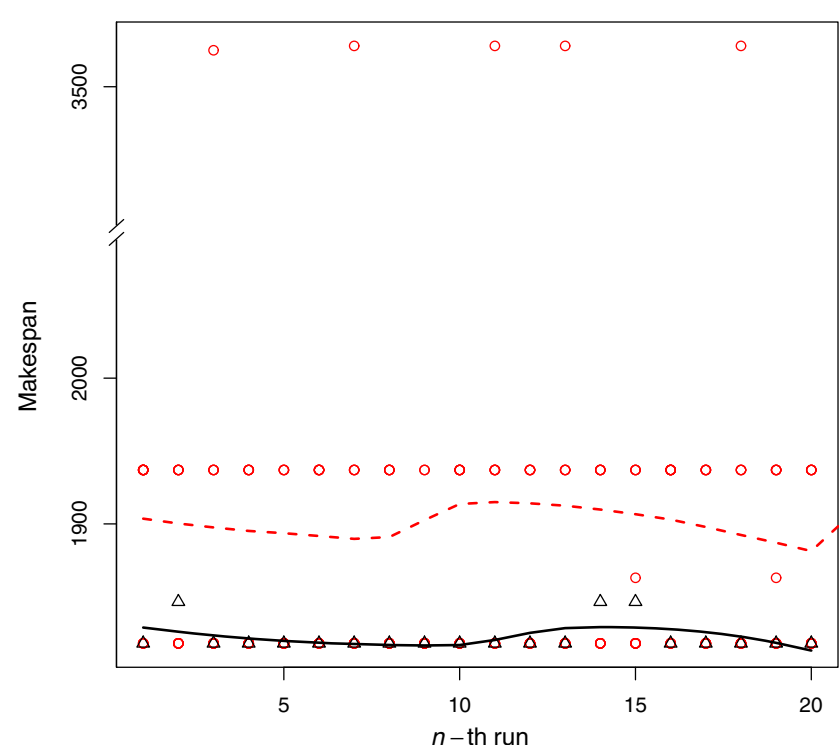

(a)

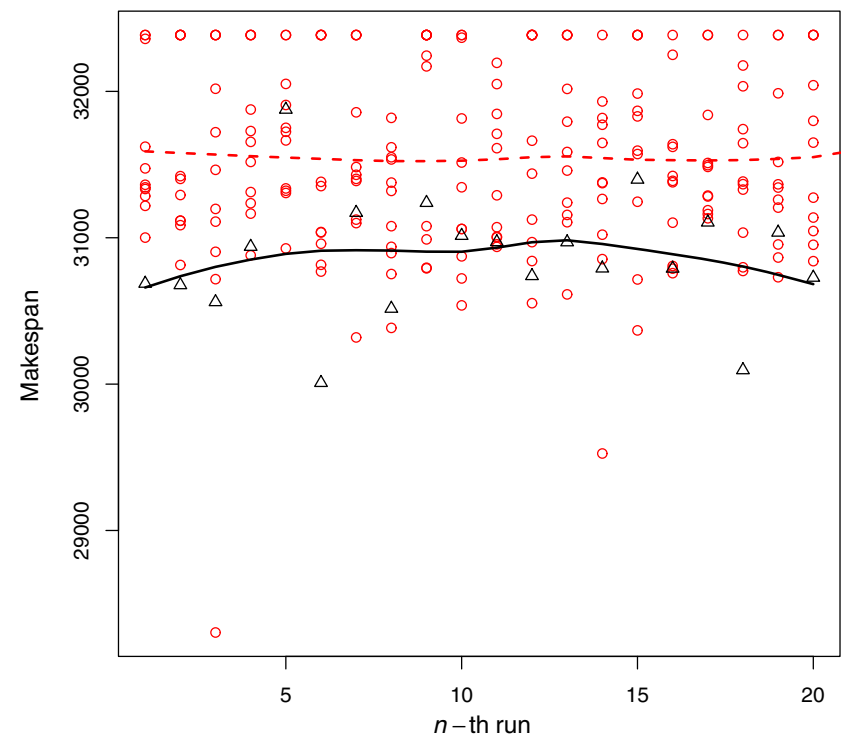

(c)

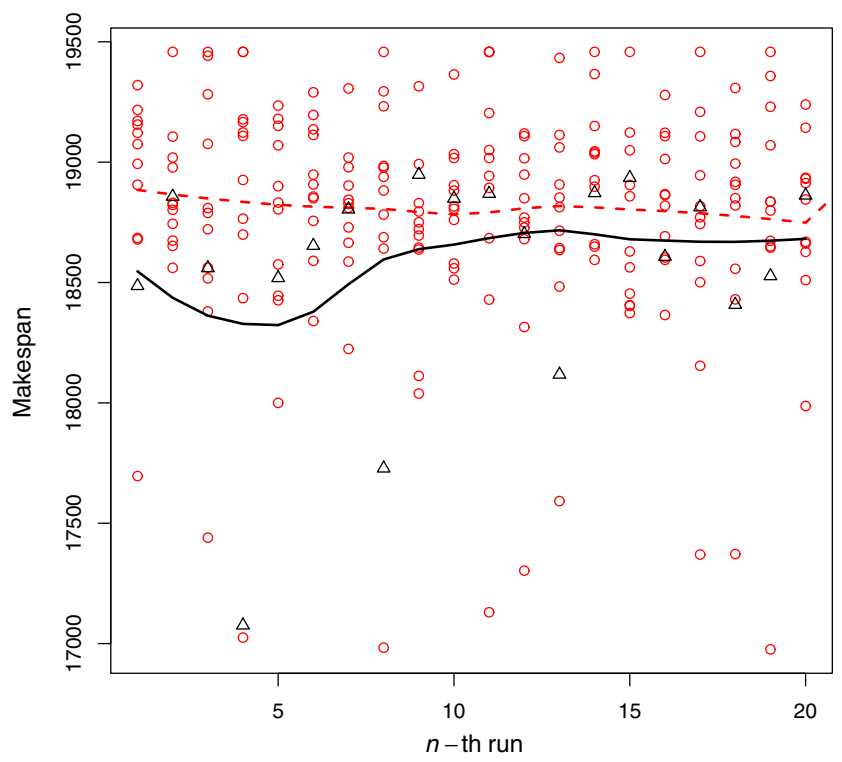

(b)

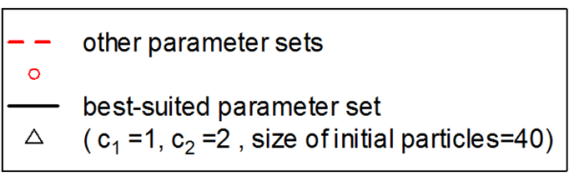

Fig. 8 Comparison of makespans from the best suited parameters against others. a Number of tasks $=10$, $\mathbf{b}$ number of tasks $=50$, $\mathbf{c}$ number of tasks $=100$

parameters $1 \leq c_{1} \leq 2$ and $1 \leq c_{2} \leq 2$. It is deduced that the best suited number of initial particles is 40 .

2. Makespan leans towards the lowest level as parameter $c_{2}$ is set to be greater than $c_{1}$ Figure 7 depicts the overall (representing results from 8, 20, and 40 initial particles) makespans from four combinations of $c_{1}$ and $c_{2}$ values. Each overall makespan is obtained through applying a local polynomial regression fitting [11] (with $\operatorname{span}=0.75$, degree $=2$ ) on the makespan data from 60 runs, which consists of equal running portions of experiment with 8,20 , and 40 initial particles (20 runs each). Makespan line of $c_{1}=1, c_{2}=2$ is shown to be lower than others most of the time. This condition is explained by Eq. (15) where $c_{1}$ is correlated with the determination level of the particle to learn from its local best particle, while $c_{2}$ is correlated with the determination level of the particle to learn from the global best particle.

In this manner, during the iterations of the search, every particle has the determination to learn more from a particle which is recalled as the best one by all particles in the swarm. 


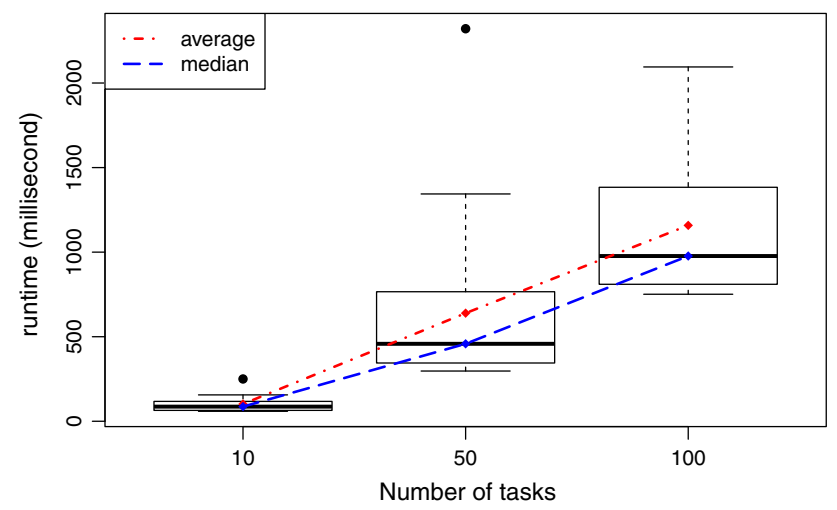

Fig. 9 Computation time of the proposed algorithm for the best suited parameters

3. Makespan tends to converge at the same convergence point when the size of the problem is small

Makespan acts as fitness value of a solution. In Fig. 13a, d, g, j, it is shown that among multiple distinct runs, a relatively common makespan is found. That means those different runs have a relatively common convergence point (of solution). It is an extreme phenomenon upon the benefit of having highly spread initial particles in the beginning of the search. Moreover, since the number of possible solutions is relatively not high, nearly all local optimum points are visited, leading to a high chance of finding a global optimum solution which is the same for all runs.

Consequently, the trade-off relationships among PSO parameters are depicted in Table 10.

\subsubsection{Performance evaluation}

Previously, in Fig. 13, the optimality level evaluation for several sets of parameters has been addressed. Afterwards, convergence speed evaluation of each set of parameter is shown, respectively, in Fig. 14. This convergence evaluation is done by checking the value of makespan from the obtained solution. Once there is no improvement after 10 contiguous iterations, the search is concluded to be converged at that particular iteration. For all combinations, most of the test cases converged before iteration 40 . For the best case of interest so far, where $c_{1}=1, c_{2}=2$, and the number of initial particles equals to 40 , the search managed to converge under iteration 25 . The ultimate goal is to cut the excessive number of iterations if the search is proven to most likely converge only after less than the originally given one. Hence, one might think about cutting the number of iterations to 30 because even with this limitation, the search is able to bring a good feasible schedule for the given dataset. However, for a marginal tolerance, the maximum number of iterations may still be set to 40 , the highest number of iterations so far from all searches. In this manner, it still gives some space for the search to explore more if it is trapped in some local optimum points in the beginning of the search.

\section{Results and discussion}

From the numerical experiment, the best suited set of parameters for the search is selected: $c_{1}=1, c_{2}=2$, number of initial particles $=40$. In addition, based on a numerous experiments, the maximum number of iterations $=40$ provides a good room for the search to progress, while higher values are often not worth the increased computation time. The result is shown in Fig. 8; the graphs are distinguished by the number of tasks. Each graph represents points and line (with local polynomial regression fitting) plot of makespans obtained from search which uses the best suited set of parameters (represented by triangle points and full line) against others (represented by circle points and dashed line), with composition of 20:220, respectively, resulting in total of 240 makespans plotted in each graph. The way the data plotted on $y$-axis is based on the respective makespan values. As for $x$-axis, it is based on the run index values; as mentioned in 5 , each set of parameters is run for 20 times.

In reference with the data shown in Fig. 13, a numerical summary of the experiments with the best suited parameters on three task datasets is depicted in Table 11. The presented numbers are in accordance with the makespans obtained from the best suited parameters, which are displayed in Fig. 8. Both (lines in) Fig. 8 and Table 11 can show a relatively small variance of makespans, which indicates a stable performance of the best suited parameters.
Table 11 Numerical summary of experiments with the best suited parameters

\begin{tabular}{|c|c|c|c|c|c|c|c|}
\hline \multicolumn{3}{|c|}{ Parameter } & \multirow[t]{2}{*}{ Number of tasks } & \multicolumn{4}{|c|}{ Makespan (ms) } \\
\hline$c_{1}$ & $c_{2}$ & Number of initial particles & & Min & Max & Average & Median \\
\hline \multirow[t]{3}{*}{1} & 2 & 40 & 10 & 1818 & 1937 & 1835.85 & 1818 \\
\hline & & & 50 & 17076 & 18948 & 18559.65 & 18677.5 \\
\hline & & & 100 & 30009 & 31876 & 30865.65 & 30865 \\
\hline
\end{tabular}


Furthermore, a computation time measurement of the best suited parameters is depicted in Fig. 9. The dotteddashed line in Fig. 9 depicts the average computation time, while the dashed line depicts the median of the makespan throughout the conducted experiments. It is shown that as the number of tasks scales $(10,50,100)$, the computation time tends to scale linearly (it varies around an average of $102.1,639.25$, and $1158.25 \mathrm{~ms}$, respectively). It is possible to see that the presented methodology is efficient because during those short computation times, it can obtain a set of schedules and choose the best one through a number of iterations, conducting a solid optimization. Each box plot of the task dataset represents 20 computation times. There is one computation time observation of $2321 \mathrm{~ms}$ on the schedule generation of 20 tasks, which is obviously far from the average. Hence, it can be inferred as an outlier, which is bound to occur in metaheuristic.

\section{Conclusion}

Indoor UAV application has been emerging in various application domains, such as manufacturing environment. It exposes a number of new benefits and challenges simultaneously. An autonomous UAV operation reduces the number of human interventions, but it certainly requires a precise UAV flight control and scheduling system. In connection with the considered problem, a mathematical model is presented. To solve this, a methodology which controls the execution of tasks by UAV over a period of time to achieve a minimum total makespan is developed. A heuristic (based on earliest available time algorithm) for assigning tasks to UAVs which forms a seamless UAV operations schedule (considering the required recharge, hover, and wait-on-ground) is proposed. This heuristic is incorporated with particle swarm optimization (PSO) algorithm which promotes a short computation time in finding a high-quality feasible solution. The implementation of the proposed methodology solves the presented problem, which has not been done so far in the reported literature. Numerical experiments are conducted to analyse the behaviour of PSO parameters and select the best suited set. The performance of the implemented methodology is also measured through its convergence speed and computation time. The obtained results are presented and discussed in detail.

The proposed work is a pilot study of task scheduling system for UAV operations in indoor environment. It serves as a foundation for further extensions in the future work. In the future work, anti-collision refinement phase will be incorporated with the presented system. In addition, the performance of current methodology can be compared with other well-known metaheuristics reported in the literature. A more detailed study on the distributed control of UAVs can be conducted, where a seamless communication among the UAVs plays an important role. In the study of this distributed approach, the robustness of the respective system can be assessed.

Acknowledgements This work has partly been supported by Innovation Fund Denmark under project UAWorld; grant agreement number 9-2014-3.

\section{Compliance with ethical standards}

Conflict of Interest The authors declare that they have no conflict of interest.

Open Access This article is distributed under the terms of the Creative Commons Attribution 4.0 International License (http://creative commons.org/licenses/by/4.0/), which permits unrestricted use, distribution, and reproduction in any medium, provided you give appropriate credit to the original author(s) and the source, provide a link to the Creative Commons license, and indicate if changes were made.

\section{Appendix}

\section{Illustrative usage of Algorithm 2 (steps 3-7) on task sequence in Figure 4}

See Figs. 10, 11 and 12.
Fig. 10 Output of step 3 of the schedule creation heuristic

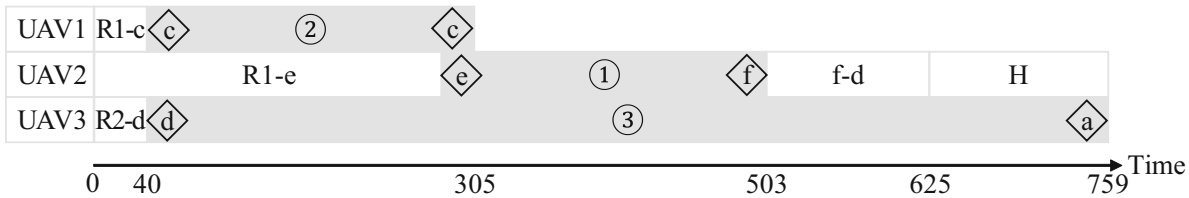


Fig. 11 Output of steps $4-6$ of the schedule creation heuristic

\begin{tabular}{|c|c|}
\hline$\frac{\text { Step }}{3}$ & Description \\
\hline & (a) Task availability check \\
\hline & Task 1 is available from time 0. \\
\hline & (b) UAV availability check \\
\hline & UAV1 : $305+228+243+60=836$ \\
\hline & UAV2 $: 0+260+243+60=563$ \\
\hline & UAV3 $: 759+346+243+60=1408>1200$ \\
\hline & UAV3 needs a recharge. \\
\hline & (c) Overall availability check \\
\hline & UAV1 : $305+228=533$ \\
\hline & UAV2 $\quad: 0+260=260$ \\
\hline & UAV3 $: 759+40+2700+260=3759$ \\
\hline & $: 759+160+2700+60=3679$ \\
\hline & $\therefore$ UAV2 is selected for task 1 \\
\hline
\end{tabular}

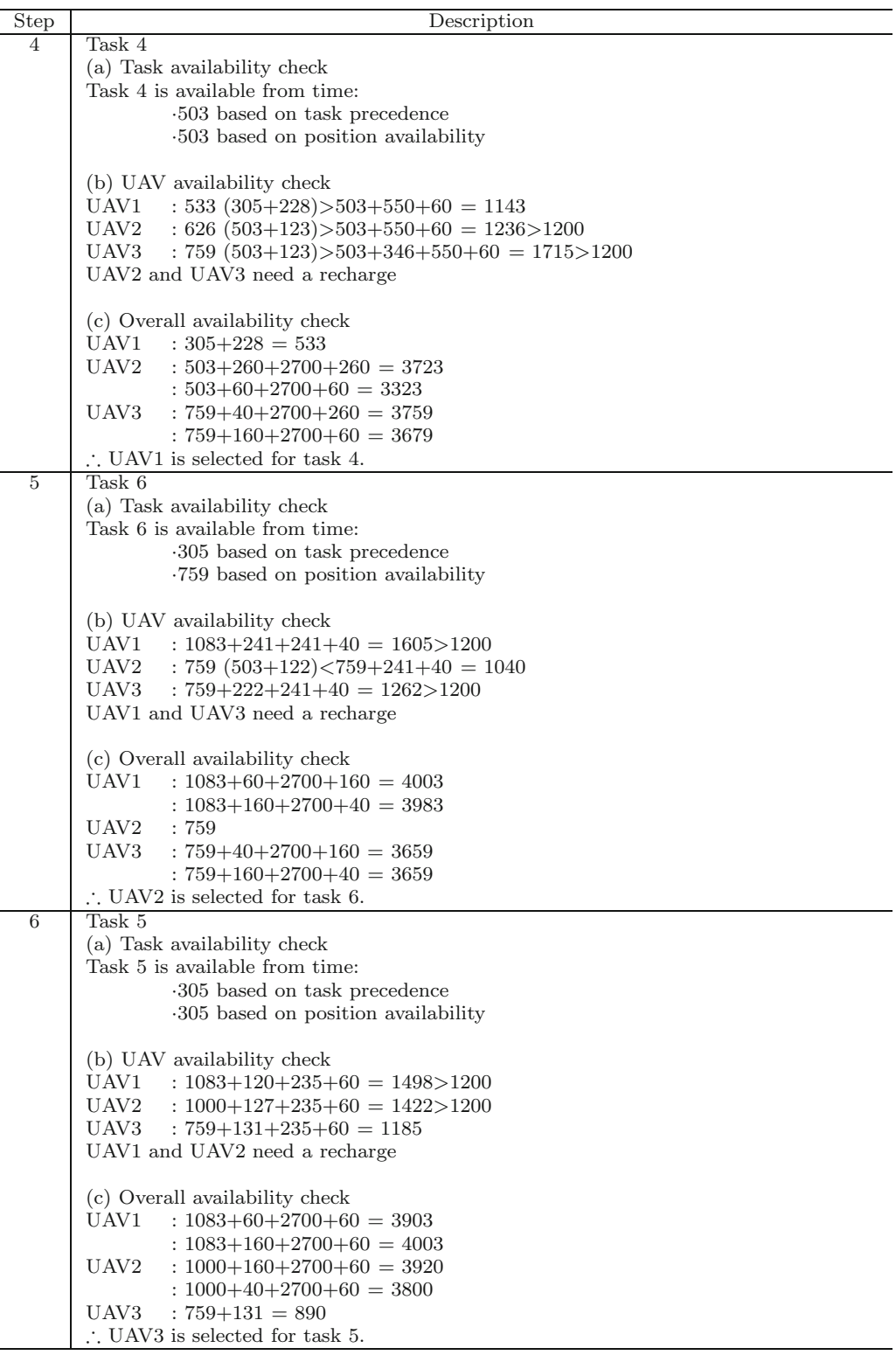


Fig. 12 Output of step 7 of the schedule creation heuristic

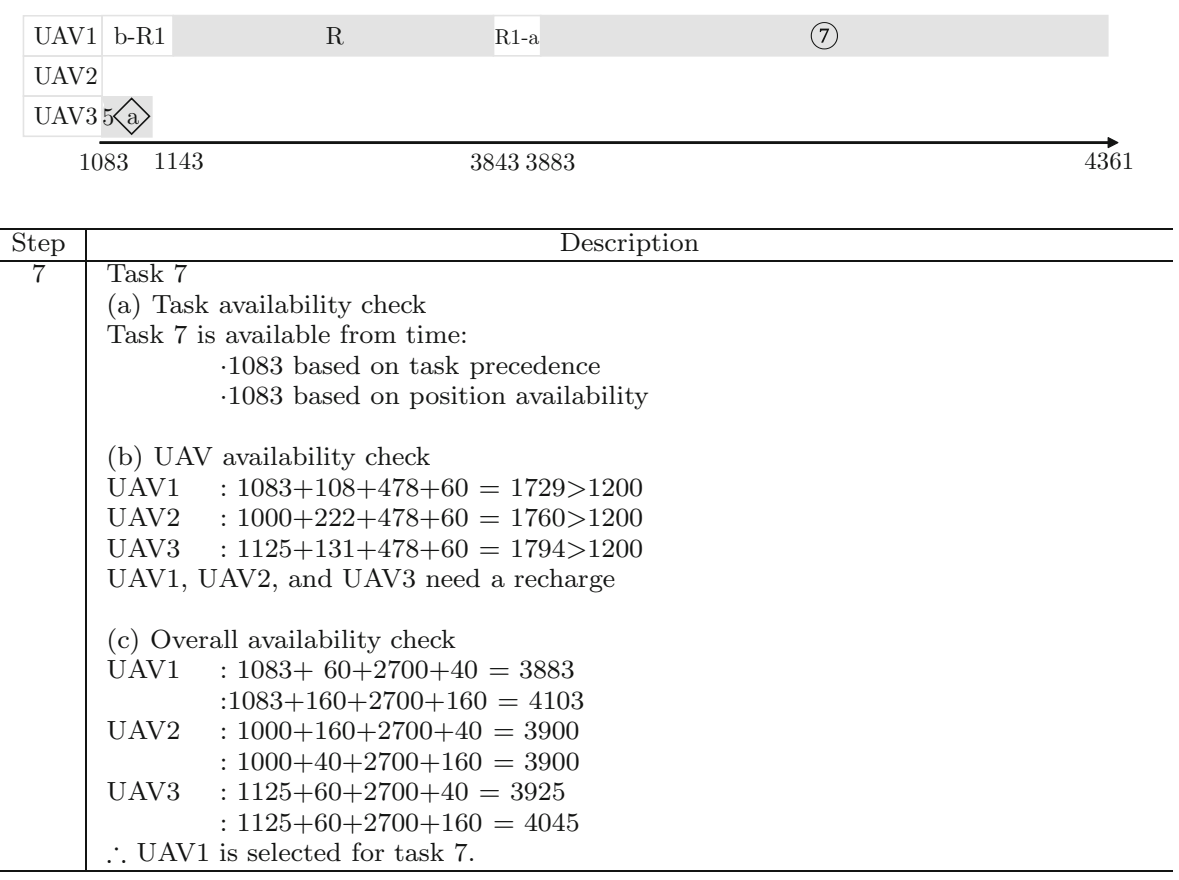

Makespans of the generated schedules based on various combinations of parameter valuesincluding $c_{1}, c_{2}$, and number of initial particleson task datasets which contain 10, 50, and 100 tasks

Each graph in Fig. 13, for instance Fig. 13a, presents the result of 60 experimental runs. For every set of initial particles: 8,20 , and 40 , the corresponding 20 makespans are represented as a box plot. This box plot provides a quick way to examine the distribution of the values, which is mainly depicted through its quartiles $(\mathrm{Q} 1$, median-Q2, Q3). While the box plot provides a more detailed information, an overlaid dotted-dashed line which depicts the mean of the numerical group gives a more general view of the data. 


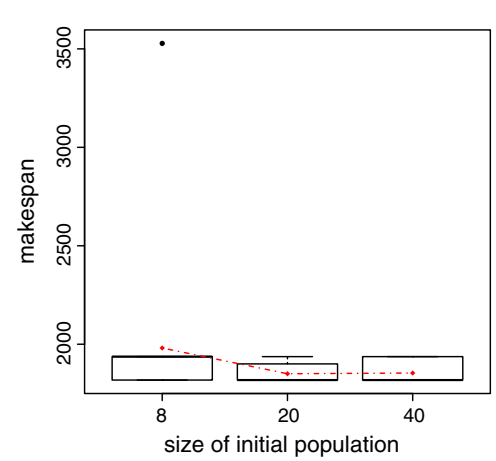

(a)

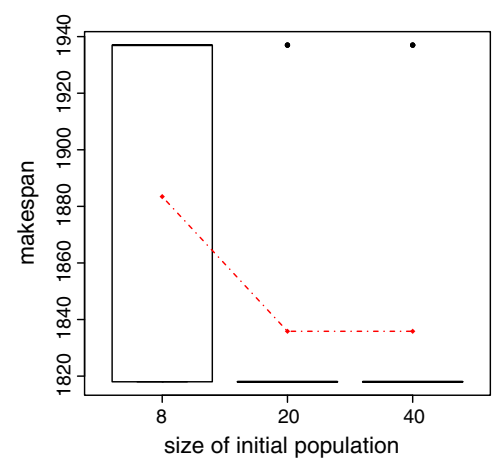

(d)

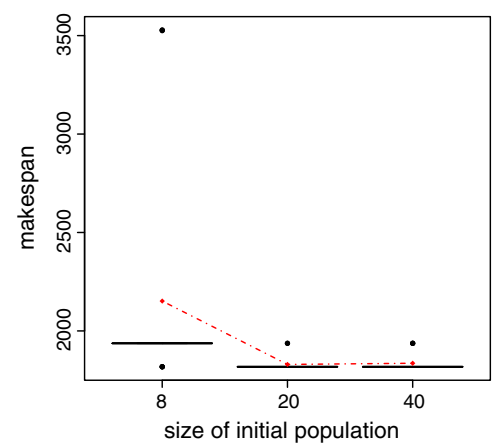

(g)

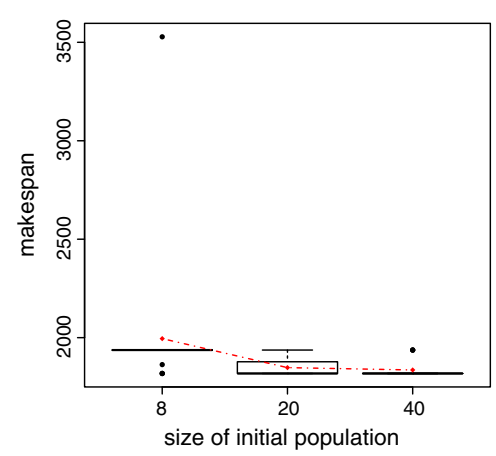

(j)

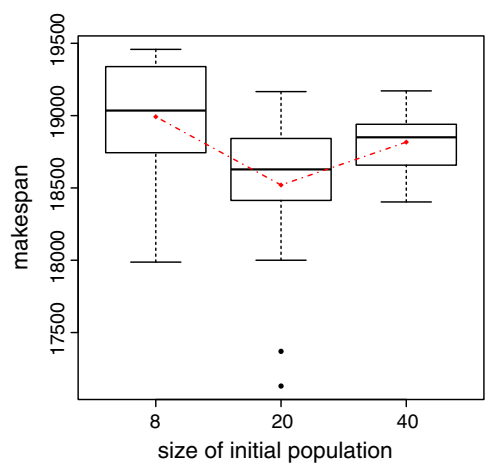

(b)

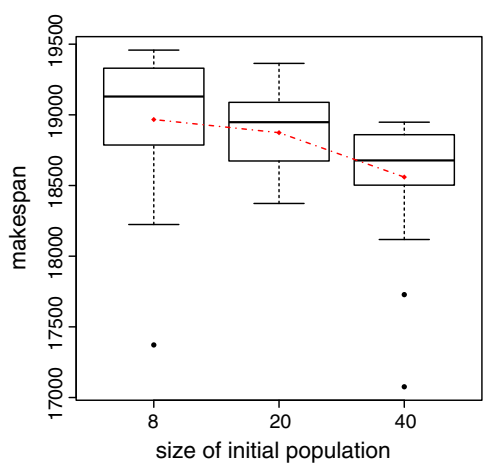

(e)

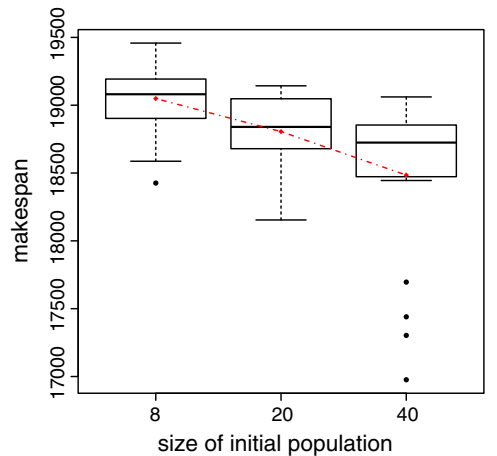

(h)

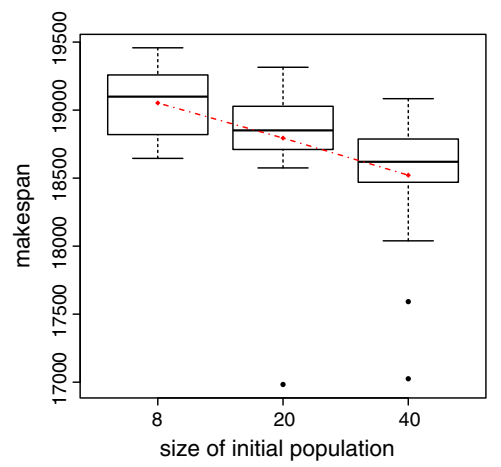

(k)

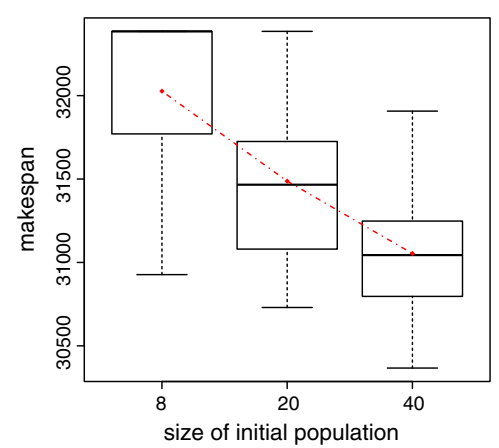

(c)

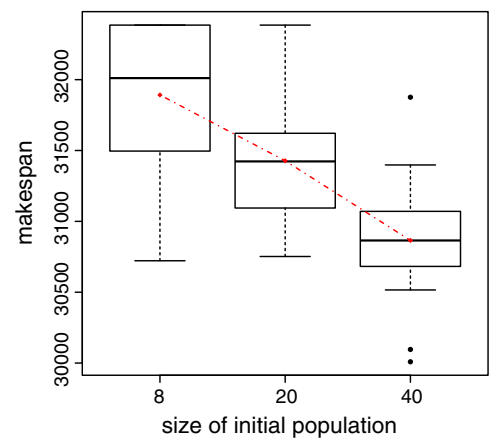

(f)

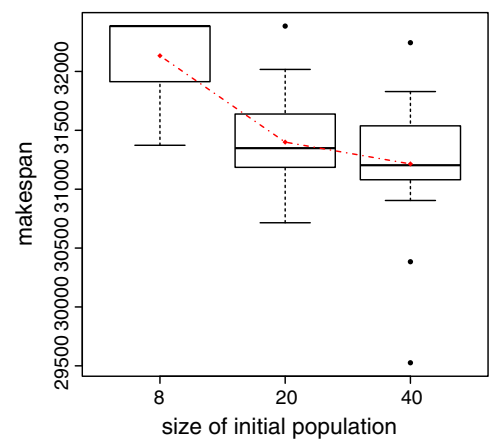

(i)

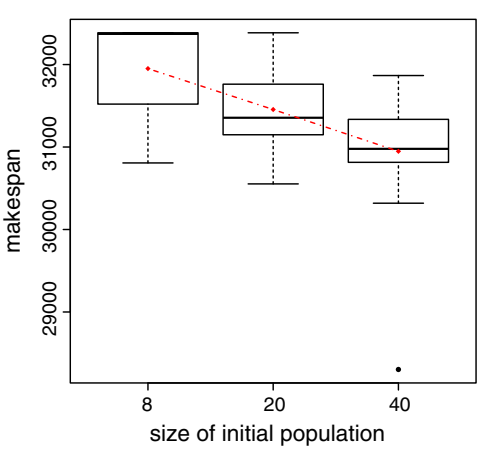

(1)
Fig. 13 Learning coefficients $\left(c_{1}\right.$ and $\left.c_{2}\right)$ analysis. a $c_{1}=1, c_{2}=1$, number of tasks $=10 ; \mathbf{b} c_{1}=1, c_{2}=1$, number of tasks $=50$; $\mathbf{c}$ $c_{1}=1, c_{2}=1$, number of tasks $=100 ; \mathbf{d} c_{1}=1, c_{2}=2$, number of tasks $=10 ; \mathbf{e} c_{1}=1, c_{2}=2$, number of tasks $=50 ; \mathbf{f} c_{1}=1, c_{2}=2$, number of tasks $=100 ; \mathbf{g} c_{1}=2, c_{2}=1$, number of tasks $=10 ; \mathbf{h}$ $c_{1}=2, c_{2}=1$, number of tasks $=50 ; \mathbf{i} c_{1}=2, c_{2}=1$, number of tasks $=100 ; \mathbf{j} c_{1}=2, c_{2}=2$, number of tasks $=10 ; \mathbf{k} c_{1}=2$, $c_{2}=2$, number of tasks $=50 ; \mathbf{l} c_{1}=2, c_{2}=2$, number of tasks $=100$ 
Convergence speed of the search with various combinations of parameter values-including $c_{1}$, $c_{2}$, and number of initial particles-on task datasets which contain 10, 50, and 100 tasks

Each graph in Fig. 14, for instance Fig. 14a, presents the result of 60 experimental runs. For every set of initial particles: 8, 20 and 40, the corresponding 20 convergence speeds are represented as a box plot. This box plot provides a quick way to examine the distribution of the values, which is mainly depicted through its quartiles (Q1, median-Q2, Q3). While the box plot provides a more detailed information, an overlayed dotted-dashed line which depicts the mean of the numerical group gives a more general view of the data. 


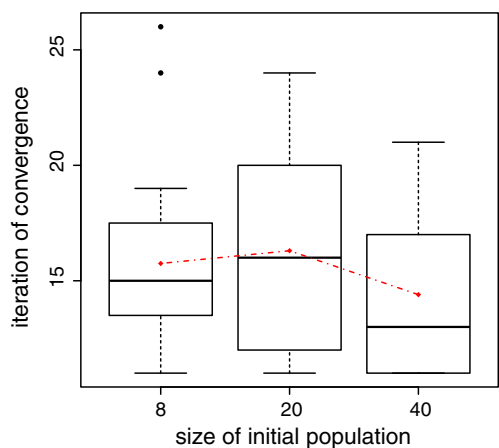

(a)

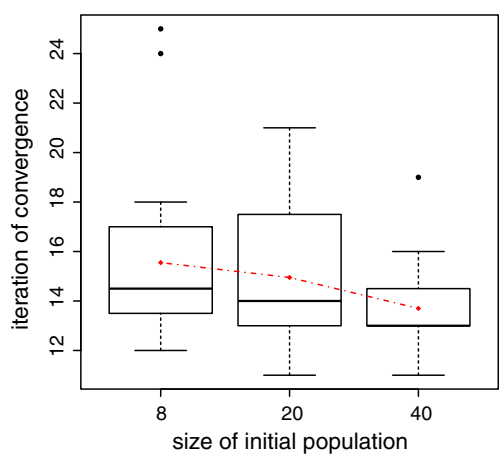

(d)

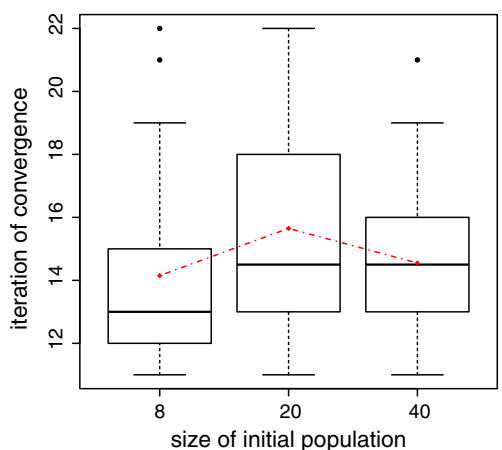

(g)

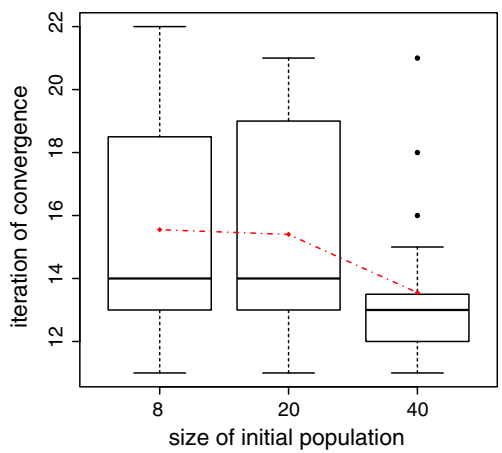

(j)

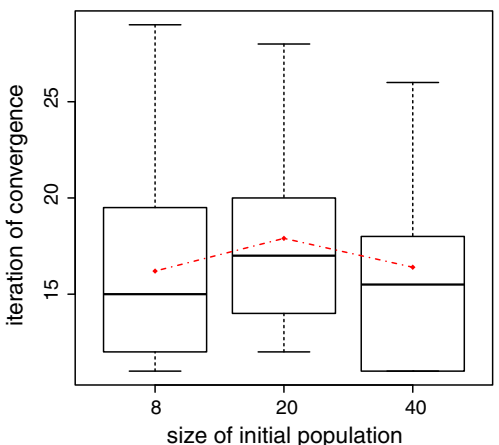

(b)

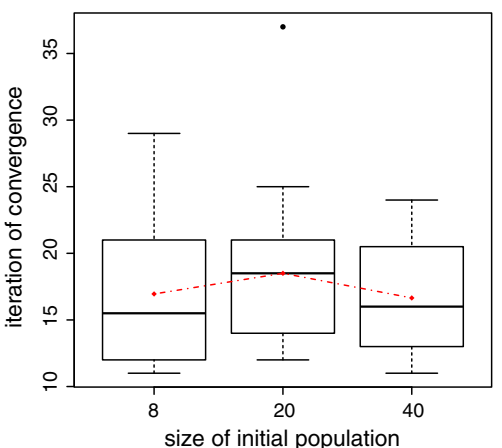

(e)

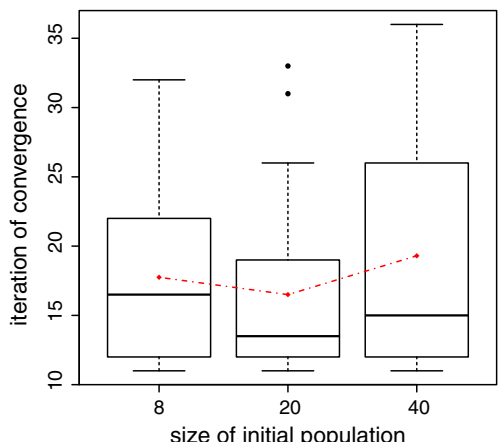

(h)

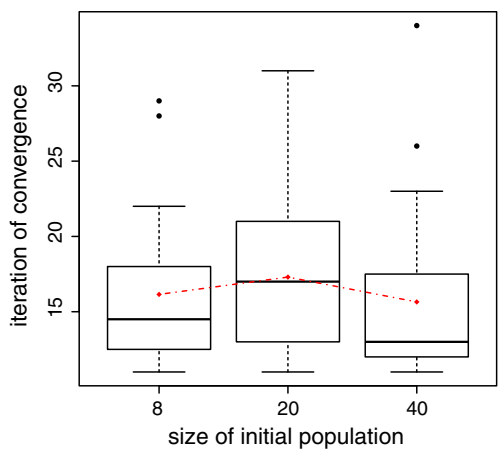

(k)

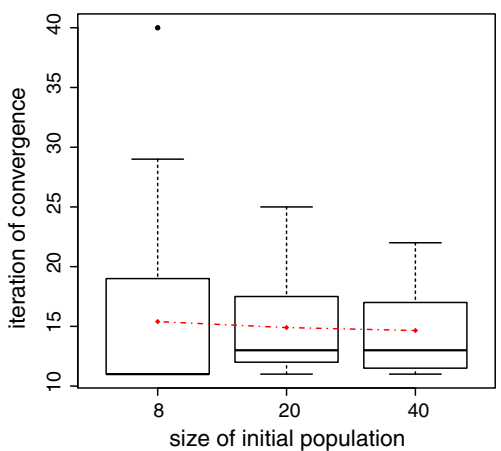

(c)

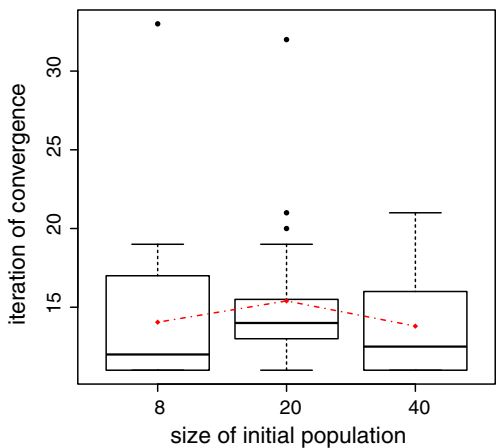

(f)

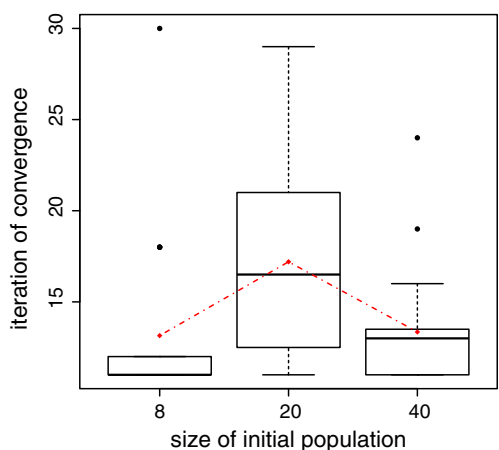

(i)

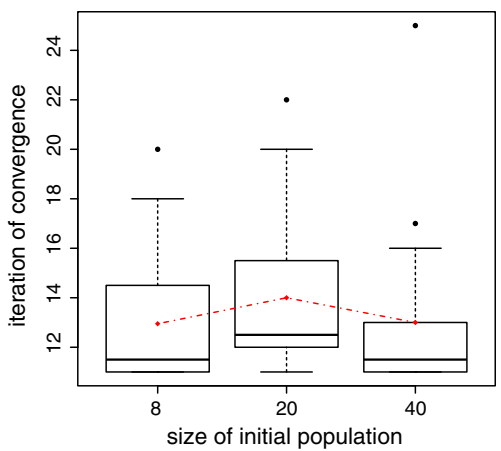

(I)
Fig. 14 Convergence speed analysis. a $c_{1}=1, c_{2}=1$, number of tasks $=10 ; \mathbf{b} \quad c_{1}=1, c_{2}=1$, number of tasks $=50 ; \mathbf{c} c_{1}=1$, $c_{2}=1$, number of tasks $=100 ; \mathbf{d} c_{1}=1, c_{2}=2$, number of tasks $=10 ; \mathbf{e} c_{1}=1, c_{2}=2$, number of tasks $=50 ; \mathbf{f} c_{1}=1$, $c_{2}=2$, number of tasks $=100 ; \mathbf{g} c_{1}=2, c_{2}=1$, number of tasks $=10 ; \quad \mathbf{h} \quad c_{1}=2, \quad c_{2}=1$, number of tasks $=50 ; \mathbf{i} \quad c_{1}=2$, $c_{2}=1$, number of tasks $=100 ; \mathbf{j} c_{1}=2, c_{2}=2$, number of tasks $=10 ; \mathbf{k} \quad c_{1}=2, c_{2}=2$, number of tasks $=50 ; \mathbf{l} c_{1}=2$, $c_{2}=2$, number of tasks $=100$ 


\section{References}

1. Ahner DK, Buss AH, Ruck J (2006) Assignment scheduling capability for unmanned aerial vehicles: a discrete event simulation with optimization in the loop approach to solving a scheduling problem. In: Proceedings of the 38th conference on winter simulation. Winter Simulation Conference, pp 1349-1356

2. Akturk MS, Yilmaz H (1996) Scheduling of automated guided vehicles in a decision making hierarchy. Int $\mathrm{J}$ Prod Res 34(2):577-591

3. Alidaee B, Wang H, Landram F (2009) A note on integer programming formulations of the real-time optimal scheduling and flight path selection of UAVs. IEEE Trans Control Syst Technol 17(4):839-843

4. Tijani IB, Akmeliawati R, Legowo A, Budiyono A, Abdul Muthalif AG (2014) Hybrid DE-PEM algorithm for identification of UAV helicopter. Aircr Eng Aerosp Technol Int J 86(5):385-405

5. Bethke B, Valenti M, How JP (2008) UAV task assignment. IEEE Robot Autom Mag 15(1):39-44

6. Błażewicz J, Domschke W, Pesch E (1996) The job shop scheduling problem: conventional and new solution techniques. Eur J Oper Res 93(1):1-33

7. Blondin J (2009) Particle swarm optimization: a tutorial. http://cs. armstrong.edu/saad/csci8100/pso_tutorial.pdf

8. Boucher P (2015) Domesticating the drone: the demilitarisation of unmanned aircraft for civil markets. Sci Engineering Ethics 21(6):1393-1412

9. Budiyono A, Lee G, Kim GB, Park J, Kang T, Yoon KJ (2015) Control system design of a quad-rotor with collision detection. Aircr Eng Aerosp Technol Int J 87(1):59-66

10. Choi BK, Kang DH (2013) Modeling and simulation of discrete event systems. Wiley, Hoboken

11. Cleveland WS, Grosse E, Shyu WM (1992) Statistical models. In: Local regression models. Wadsworth \& Brooks/Cole, Chapter 8

12. Edis EB, Oguz C, Ozkarahan I (2013) Parallel machine scheduling with additional resources: notation, classification, models and solution methods. Eur J Oper Res 230(3):449-463

13. Fasano G, Accardo D, Moccia A, Carbone C, Ciniglio U, Corraro F, Luongo S (2008) Multi-sensor-based fully autonomous noncooperative collision avoidance system for unmanned air vehicles. J Aerosp Comput Inf Commun 5(10):338-360

14. Garey MR, Johnson DS (1979) A guide to the theory of NPcompleteness. WH Freemann, New York

15. Garey MR, Johnson DS (2002) Computers and intractability, vol 29. WH Freeman, New York

16. Garrido A, Salido MA, Barber F, López M (2000) Heuristic methods for solving job-shop scheduling problems. In: Proceedings of the ECAI-2000 workshop on new results in planning, scheduling and design (PuK2000). pp 44-49

17. Gunter CA, Gunter EL, Jackson M, Zave P (2000) A reference model for requirements and specifications. In: Proceedings of the 4th international conference on requirements engineering. IEEE, pp 189

18. Hassan R, Cohanim B, De Weck O (2005) Venter Gerhard A comparison of particle swarm optimization and the genetic algorithm. In: Proceedings of the 1st AIAA multidisciplinary design optimization specialist conference. pp 1-13

19. Hou Z-L, Guo X-P (2013) Parallel machine scheduling with resources constraint and sequence dependent setup times. In: Proceedings of 2012 3rd International Asia Conference on Industrial Engineering and Management Innovation (IEMI2012). Springer, pp 801-811
20. Kellerer H, Strusevich VA (2008) Scheduling parallel dedicated machines with the speeding-up resource. Nav Res Logist 55(5):377-389

21. Kennedy J (2010) Particle swarm optimization. In: Encyclopedia of machine learning. Springer, pp 760-766

22. Khosiawan Y, Nielsen I (2016) A system of UAV application in indoor environment. Prod Manuf Res 4(1):2-22

23. Khosiawan Y, Nielsen I, Do NAD, Yahya BN (2016) Concept of indoor 3d-route UAV scheduling system. In: Information systems architecture and technology: proceedings of 36th international conference on information systems architecture and technologyISAT 2015-part I. Springer, pp 29-40

24. Jonghoe Kim, Morrison James R (2014) On the concerted design and scheduling of multiple resources for persistent UAV operations. J Intell Robot Syst 74(1-2):479-498

25. Kim J, Song BD, Morrison JR (2013) On the scheduling of systems of UAVs and fuel service stations for long-term mission fulfillment. J Intell Robot Syst 70(1-4):347-359

26. Kim Y, Gu D-W, Postlethwaite I (2007) Real-time optimal mission scheduling and flight path selection. IEEE Trans Automat Control 52(6):1119-1123

27. Liu Z (2007) Investigation of particle swarm optimization for job shop scheduling problem. In: Third International Conference on Natural Computation, ICNC 2007, volume 3. IEEE, pp 799-803

28. Iwan Mahmud, Rini Akmeliawati, Agus Budiyono (2014) DEbased robust controller design for helicopter cruise control. Int $\mathbf{J}$ Robot Mechatron 1(4):145-151

29. Mitchell JE (2002) Branch-and-cut algorithms for combinatorial optimization problems. Handb Appl Optim 2:65-77

30. Nigam N, Bieniawski S, Kroo I, Vian J (2012) Control of multiple UAVs for persistent surveillance: algorithm and flight test results. IEEE Trans Control Syst Technol 20(5):1236-1251

31. Nilakantan JM, Ponnambalam SG, Jawahar N, Kanagaraj G (2015) Bio-inspired search algorithms to solve robotic assembly line balancing problems. Neural Comput Appl 26(6):1379-1393

32. Park Y, Khosiawan Y, Moon I, Janardhanan MN, Nielsen I (2016) Scheduling system for multiple unmanned aerial vehicles in indoor environments using the CSP approach. In: Intelligent decision technologies 2016. Springer, pp 77-87

33. Piciarelli C, Micheloni C, Martinel N, Vernier M, Foresti GL (2013) Outdoor environment monitoring with unmanned aerial vehicles. In: International Conference on Image Analysis and Processing-ICIAP 2013. Springer, pp 279-287

34. Ponnambalam SG, Aravindan P, Naidu GM (2000) A multi-objective genetic algorithm for solving assembly line balancing problem. Int J Adv Manuf Technol 16(5):341-352

35. Qi X, Chen T, Tu F (1999) Scheduling the maintenance on a single machine. J Oper Res Soc 50(10):1071-1078

36. Rameshkumar K, Suresh RK, Mohanasundaram KM (2005) Discrete particle swarm optimization (DPSO) algorithm for permutation flowshop scheduling to minimize makespan. In: International conference on natural computation. Springer, pp 572-581

37. Rothkopf MH (1966) Scheduling independent tasks on parallel processors. Manag Sci 12(5):437-447

38. Semiz F (2015) Task assignment and scheduling in UAV mission planning with multiple constraints. Ph.D. Thesis, Middle East Technical University

39. Sha DY, Hsu C-Y (2006) A hybrid particle swarm optimization for job shop scheduling problem. Comput Ind Eng 51(4):791-808

40. Sha DY, Lin H-H (2010) A multi-objective pso for job-shop scheduling problems. Expert Syst Appl 37(2):1065-1070

41. Shaw P (2004) A constraint for bin packing. In: International conference on principles and practice of constraint programming. pp 648-662 
42. Shima T, Rasmussen SJ, Sparks AG (2005) UAV cooperative multiple task assignments using genetic algorithms. In: American Control Conference, 2005. Proceedings of the 2005. IEEE, pp 2989-2994

43. Shima T, Schumacher C (2005) Assignment of cooperating UAVs to simultaneous tasks using genetic algorithms. Defense Technical Information Center, San Francisco, California

44. Sörensen K, Glover FW (2013) Metaheuristics. In: Encyclopedia of operations research and management science. Springer, pp 960-970

45. Tijani IB, Akmeliawati R, Legowo A, Budiyono A (2014) Nonlinear identification of a small scale unmanned helicopter using optimized NARX network with multiobjective differential evolution. Eng Appl Artif Intell 33:99-115

46. Valenti M, Dale D, How J, Vian J (2007) Mission health management for $24 / 7$ persistent surveillance operations. In: AIAA guidance, control and navigation conference. Myrtle Beach, SC
47. von Bueren SK, Burkart A, Hueni A, Rascher U, Tuohy MP, Yule IJ (2015) Deploying four optical uav-based sensors over grassland: challenges and limitations. Biogeosciences 12(1):163-175

48. Weinstein AL, Schumacher C (2007) UAV scheduling via the vehicle routing problem with time windows. In: Proceedings of the AIAA infotech@ aerospace 2007 conference and exhibit. Rohnert Park, California

49. Xu D, Sun K, Li H (2008) Parallel machine scheduling with almost periodic maintenance and non-preemptive jobs to minimize makespan. Comput Oper Res 35(4):1344-1349

50. Zeng J, Yang X, Yang L, Shen G (2010) Modeling for UAV resource scheduling under mission synchronization. J Syst Eng Electron 21(5):821-826

51. Zobolas GI, Tarantilis CD, Ioannou G (2008) Exact, heuristic and meta-heuristic algorithms for solving shop scheduling problems. In: Metaheuristics for scheduling in industrial and manufacturing applications. Springer, pp 1-40 